\title{
FREE FERMIONS IN TWO-DIMENSIONAL ISING MODEL
}

\author{
V. N. Plechko \\ Bogoliubov Laboratory of Theoretical Physics, Joint Institute for Nuclear Research, \\ 141980, Dubna, Moscow Region, Russia
}

(Received August 26, 1999)

\begin{abstract}
The two-dimensional (2D) Ising model is reviewed as a theory of free fermions on a lattice. The discussion includes the fermionization procedure based on the mirror-ordered factorization of the density matrix, Gaussian fermionic integral representation for partition function, the momentumspace analysis and Onsager's result, the effective continuum-limit field theories and the criticalpoint singularities. The emergence of long-range fermionic correlations in a nonzero magnetic field and the behaviour of the specific heat along the critical isotherm are commented. Attention is given to the choice of rational computational devices.
\end{abstract}

Key words: Ising model, anticommuting Grassmann variables, critical point, fermions.

PACS numbers: 64.60.Cn; 75.10.-b; 05.30.Fk

\section{INTRODUCTION}

There are many remarkable analogues between the physical concepts and mathematical methods in statistical mechanics and quantum field theory. The twodimensional Ising model (2DIM) may be a good example of this kind. In its original formulation, the 2D Ising model is a discrete-spin lattice model for the secondorder phase transitions in magnets, for which the analytic results for the free energy and some other functions are available over the whole temperature range. At the first stages, the 2DIM has been analyzed rather as a complicated mathematical problem [1-8]. The canonical approaches to the 2DIM are based on the transfer-matrix and combinatorial considerations [1-12]. The fermionic features in the 2DIM have been recognized already in $[2,6,7]$. Further developments in this direction provided new insight into the physical nature of the problem and significantly simplified the analysis in the technical aspect $[13-20]$. The modern approaches to the 2DIM are based on the interpretation of the problem in terms of fermions $[13-26]$. In this article, we review some aspects of a simple noncombinatorial fermionic approach to the 2D Ising model based on the application of the anticommuting Grassmann-variable integrals and the mirrorordered factorization ideas for the density matrix [18-20]. The method is simple and is in a sense straightforward. The transfer-matrices and combinatorics are not used. The appearance of fermions in the $2 \mathrm{D}$ Ising model within given approach rather resembles the change of the basis in quantum mechanics. The article is arranged as follows. A short introduction to the rules of the fermionic integration is given in Section II. The original spin-variable formulation for the 2D Ising model is considered in Section III. We then directly proceed with discussing the fermionization procedure in Section IV. The Grassmann variables are first introduced by factorization of the local bond Boltzmann weights in (15). The mirror-ordered factorized representation for the whole density matrix is obtained at the next stage in (21). This is a mixed spin-fermion representation for the density matrix, in which spin variables can be readily eliminated. This results the Gaussian fermionic integral for partition function, $Q$, given in (24). Equivalently, the 2D Ising model is reformulated as a theory of free fermions on a lattice. The transformation of $Q$ into a fermionic integral is performed in Section IV for the most general inhomogeneous distribution of the bond coupling parameters over the lattice. In Section $V$ the 2D Ising model on the standard homogeneous rectangular lattice is considered. After transformation to the momentum space for fermions, the partition function is evaluated in a closed form, which results the Onsager's expression for the free energy. In Section VI, coming back to previous discussion, we add a few further remarks on the ordered products of Grassmannian factors, like those arising by factorization of the density matrix in (21). The nonlocal fermionic sums arising in this context are of interest for the 2D Ising model in a nonzero magnetic field, as is commented in Section IX. In Section VII, a refined version of the basic integral (24) for the partition function is obtained. The resulting Gaussian integral for $Q$ with two variables per site is given in (57). It is interesting that the MajoranaDirac structures, somewhat mysteriously arising in the 2D Ising model, can be recognized in the fermionic action of the integral (57) already at the lattice level. The effective continuum-limit field theories near $T_{c}$ corresponding to the low-momentum sector of the exact lattice theory, which is responsible for the critical-point singularities in the thermodynamic functions and the large-distance behaviour of the correlation functions, are considered in Section VIII. The effective Majorana like action for twocomponent massive fermions is obtained in (66). After the uniformization transformation, the action appears in the standard Majorana form given in (67) and (79). By doubling the number of fermions in the Majorana representation, one can pass as well to the Dirac theory of charged fermions (80). In Section IX, we make use of the simplified continuum-limit interpretation of 2DIM in order to discuss the critical-point singularities near $T_{c}$. The effects produced by a nonzero magnetic field in 
the fermionic system of the 2DIM Ising model near $T_{c}$ are considered within approximating approach. It is argued the that switching on of a nonzero magnetic field $(h \neq 0)$ causes the long-range nonlocal interactions of the fermions, on the backbone of the local part of the action corresponding to $h=0$. Within adopted approximation conjectures, the singularity in the specific heat at the critical isotherm is expected to be logarithmic. Finally, few concluding remarks are given in Section X. In the next section, we start with a comment on the rules of fermionic integration which are relevant for the forthcoming discussion.

\section{GRASSMANN VARIABLES}

Let us remember that Grassmann variables (nonquantum fermionic fields) are the purely anticommuting fermionic symbols. Given a set of Grassmann variables, $a_{1}, a_{2}, a_{3}, \ldots, a_{N}$, we have:

$$
a_{i} a_{j}+a_{j} a_{i}=0, \quad a_{j}^{2}=0
$$

The linear superpositions of Grassmann variables are again purely anticommuting, their squares are zeros. The first important identity of anticommuting analysis is given as follows:

$$
\begin{aligned}
& b_{1} b_{2} b_{3} \ldots b_{N}=\operatorname{det} \hat{A} \cdot a_{1} a_{2} a_{3} \ldots a_{N}, \\
& b_{i}=\sum_{j=1}^{N} A_{i j} a_{j},
\end{aligned}
$$

where we multiply the linearly transformed variables, the determinant of the matrix of the transformation appears as the coefficient between the two products. The relation of the anticommuting algebra to the determinant combinatorics expressed in the above identity is well known in physics and mathematics already for a long time. The rules of the integration for Grassmann variables have been first introduced by Berezin [27]. The elementary rules for one variable are:

$$
\int d a_{j} \cdot a_{j}=1, \quad \int d a_{j} \cdot 1=0
$$

In multiple fermionic integrals, the differential symbols are again anticommuting with each other and with the variables [27]. The integration then reduces to the repeating use of the above elementary rules, keeping in mind that the fermionic symbols anticommute. Due to the nilpotent property of fermions, $a_{j}^{2}=0$, any natural (analytic) function definite a finite set of Grassmann variables can be represented, in principle, as a finite polynomial in these variables:

$$
\begin{aligned}
f\left(a_{1}, a_{2}, \ldots, a_{N}\right) & =f_{0}+\sum_{j=1}^{N} f_{j} a_{j}+\ldots \\
& +f_{123 \ldots N} a_{1} a_{2} \ldots a_{N}
\end{aligned}
$$

where $f_{0}, f_{j}, \ldots, f_{123 \ldots N}$ are the numerical coefficients. In particular, integrating polynomial (4) according to the rules (3), we find:

$$
\int d a_{N} \ldots d a_{2} d a_{1} f\left(a_{1}, a_{2}, \ldots, a_{N}\right)=f_{123 \ldots N}
$$

The integration is thus a simple task if the integrand function is already known in the polynomial form, however, this may be not the case in applications. The rules of change of variables under a linear substitution in the fermionic integrals readily follow from (2) and (5). As compared with the rules of commuting analysis, the only difference is that the Jacobian will now appear in the inverse power [27]. In physical applications, an important role is played by Gaussian fermionic integrals. The Gaussian fermionic integral of the first kind is given as follows [27]:

$$
\int \prod_{j=1}^{N} d a_{j}^{*} d a_{j} \exp \left(\sum_{i=1}^{N} \sum_{j=1}^{N} a_{i} A_{i j} a_{j}^{*}\right)=\operatorname{det} \hat{A}
$$

where all the variables in the total set are purely anticommuting, the matrix $\hat{A}$ is arbitrary. The appearance of the determinant in (6) can be traced back to (2). By convention, the variables $a_{j}$ and $a_{j}^{*}$ can be viewed as the complex conjugated variables, in physical contexts this corresponds to charged fermions, otherwise $a_{j}$ and $a_{j}^{*}$ are simply independent variables. The Gaussian fermionic integral of the second kind, for real fermionic fields, is related to the Pfaffian [27]:

$$
\begin{aligned}
& \int d a_{N} \ldots d a_{2} d a_{1} \exp \left(\frac{1}{2} \sum_{i=1}^{N} \sum_{j=1}^{N} a_{i} A_{i j} a_{j}\right) \\
& =\operatorname{Pfaff} \hat{A} .
\end{aligned}
$$

Matrix $\hat{A}$ is now assumed to be skew-symmetric: $A+$ $A^{T}=0$, where $A^{T}$ is the transposed matrix. In components: $A_{i j}+A_{j i}=0, A_{i i}=0$. This property is complimentary to fermionic anticommutativity. By formal definition, the Pfaffian is some combinatorial polynomial in elements $A_{i j}$ known in mathematics for a long time $[28,29]$. The combinatorics of the Pfaffian is identical to that of the fermionic version of Wick's theorem $[28,29]$. Notice that the number of the variables $N$ in the integral (7) must be even, otherwise the integral is identically zero. This property is again in accordance with the formal definition of the Pfaffian with $N$ odd $[28,29]$. The equation (7) can itself be assumed for an effective defini- 


\section{N. PLECHKO}

tion of the Pfaffian, wherefrom its basic properties readily follow. For any skew-symmetric matrix, the following algebraic identity is known $[28,29]$ :

$$
\operatorname{det} \hat{A}=(\operatorname{Pfaff} \hat{A})^{2} \text {. }
$$

The Pfaffian is thus the square root of the determinant of a skew-symmetric matrix. The above identity most easily can be proved just in terms of the integrals like (6) and (7). Let $N$ be even. Assuming that the matrix in (6) is skew-symmetric, we make use of the substitution:

$$
a_{k}=\frac{1}{\sqrt{2}}\left(\xi_{k}+i \eta_{k}\right), \quad a_{k}^{*}=\frac{1}{\sqrt{2}}\left(\xi_{k}-i \eta_{k}\right)
$$

where $\xi_{k}, \eta_{k}$ are the new variables of the integration. It is then easy to check that the integral (6) decouples into a product of two identical integrals like $(7)$, which is equivalent to (8). For the normalized multifermionic averages associated with the Gaussian integrals like (6) and (7) one can apply fermionic Wick's theorem in a usual way. There are few notes in conclusion. In the fieldtheoretical language, the fermionic form in the exponents like in (6) and (7) is called action. Since the action is quadratic, the integrals (6) and (7) are Gaussian integrals. The fermionic exponents in $(6)$ and $(7)$ are to be assumed in the sense of their series expansion. Due to the nilpotent property of fermions, the exponential series definitely terminate at some stage. The above exponents are thus finite polynomials in the variables, $\mathrm{cf}$. (4).
These polynomial representations also follow by multiplying elementary exponential factors. For instance, the exponential in (6) can be viewed as a product of factors like $\exp \left(a_{i} A_{i j} a_{j}^{*}\right)=1+a_{i} A_{i j} a_{j}^{*}$. In physical interpretations, the Gaussian fermionic integrals correspond to free-fermion field theories [21,22].

\section{TWO-DIMENSIONAL ISING MODEL}

In this section the $2 \mathrm{D}$ Ising model is formulated in terms of Ising spin variables. Among the goals we keep in mind in the present exposition, one is to provide an illustration for how the analytic results can be extracted by means of Grassmann variables in Statistical Mechanics. In particular, this is realized in Section $\mathrm{V}$ when we evaluate explicitly the partition function and free energy for the 2DIM on the standard homogeneous (translationally invariant) rectangular lattice. The fermionization itself, however, can be performed equally well for the 2DIM with arbitrary distribution of the exchange energies over the lattice. So, we start here with a generalized formulation of the $2 \mathrm{D}$ Ising model, assuming arbitrary inhomogeneous distribution of the bond coupling parameters over a rectangular lattice net. The Ising spins, $\sigma_{m n}= \pm 1$, are disposed at the lattice sites, $m n$, labeled by pairs of integers, $m, n=1, \ldots, L$, with $m$ and $n$ running in horizontal and vertical directions, respectively. $L$ is the length of the lattice side. The total number of sites and spins on the lattice is $N=L^{2}$, at final stages we assume $N=L^{2} \rightarrow \infty$. The hamiltonian is:

$$
-\beta H(\sigma)=\sum_{m=1}^{L} \sum_{n=1}^{L}\left[b_{m+1 n}^{(1)} \sigma_{m n} \sigma_{m+1 n}+b_{m n+1}^{(2)} \sigma_{m n} \sigma_{m n+1}\right]
$$

where $b_{m n}^{(\alpha)}=\beta J_{m n}^{(\alpha)}$ are the dimensionless bond coupling parameters, $J_{m n}^{(\alpha)}$ are the exchange energies, $\beta=1 / k T$ is the inverse temperature in the energy units. For $L$ finite, to be definite, let us assume free boundary conditions for spin variables: $\sigma_{L+1 n}=0, \quad \sigma_{m L+1}=0$. The partition function and the free energy per site are:

$$
\begin{aligned}
& Z=\sum_{(\sigma)} \exp (-\beta H(\sigma)), \\
& -\beta f_{Z}=\lim _{N \rightarrow \infty} \frac{1}{N} \ln Z, \\
& \beta=1 / k T,
\end{aligned}
$$

where the sum in $Z$ is taken over all possible spin configurations provided by $\sigma_{m n}= \pm 1$ at each site. The internal (average) energy and specific heat follow by differentiating the free energy with respect to the temperature. The specific heat per site is: $C / k=\beta^{2} \partial^{2}\left(-\beta f_{Z}\right) / \partial \beta^{2}$, $\beta=1 / k T$, where $C / k$ is the dimensionless specific heat, $k$ is Boltzmann's constant. For a typical bond Boltzmann weight from (11), let us note the identity: $\exp \left(b \sigma \sigma^{\prime}\right)=$ $\cosh b+\sigma \sigma^{\prime} \sinh b$, which readily follows from $\sigma \sigma^{\prime}= \pm 1$. The partition function then appears in the form:

$$
Z=\left\{\prod_{m=1}^{L} \prod_{n=1}^{L}\left(2 \cosh b_{m+1 n}^{(1)} \cosh b_{m n+1}^{(2)}\right\} Q\right.
$$

where $Q$ is the reduced partition function: 


$$
Q=\operatorname{Sp}_{(\sigma)}\left\{\prod_{m=1}^{L} \prod_{n=1}^{L}\left(1+t_{m+1 n}^{(1)} \sigma_{m n} \sigma_{m+1 n}\right)\left(1+t_{m n+1}^{(2)} \sigma_{m n} \sigma_{m n+1}\right)\right\}
$$

with $t_{m n}^{(1,2)}=\tanh b_{m n}^{(1,2)}$, and now we assume a properly normalized spin averaging:

$$
\underset{(\sigma)}{\operatorname{Sp}}(\ldots)=\prod_{m n} \operatorname{Sp}_{\left(\sigma_{m n}\right)}(\ldots), \quad \underset{\left(\sigma_{m n}\right)}{\operatorname{Sp}}(\ldots)=\frac{1}{2} \sum_{\sigma_{m n}= \pm 1}(\ldots)
$$

so that at each site $\operatorname{Sp}(1)=1, \operatorname{Sp}\left(\sigma_{m n}\right)=0$. The reduced partition function $Q$ will be the main subject of our interest in what follows.

\section{FERMIONIZATION}

In this section we transform $Q$ into a fermionic Gaussian integral. The method is based on the mirror-ordered factorization procedure for the density matrix [18-20]. Let us start with a factorization of the local bond Boltzmann weights from (13). For the whole lattice, we introduce a set of the purely anticommuting Grassmann variables, $a_{m n}, a_{m n}^{*}, b_{m n}, b_{m n}^{*}$, a pair per bond, and write:

$$
\begin{aligned}
1+t_{m+1 n}^{(1)} \sigma_{m n} \sigma_{m+1 n} & =\int d a_{m n}^{*} d a_{m n} \mathrm{e}^{a_{m n} a_{m n}^{*}\left(1+a_{m n} \sigma_{m n}\right)\left(1+t_{m+1 n}^{(1)} a_{m n}^{*} \sigma_{m+1 n}\right)} \\
& =\underset{\left(a_{m n}\right)}{\operatorname{Sp}}\left\{A_{m n} A_{m+1 n}^{*}\right\}, \\
1+t_{m n+1}^{(2)} \sigma_{m n} \sigma_{m n+1} & =\int d b_{m n}^{*} d b_{m n} \mathrm{e}^{b_{m n} b_{m n}^{*}\left(1+b_{m n} \sigma_{m n}\right)\left(1+t_{m n+1}^{(2)} b_{m n}^{*} \sigma_{m n+1}\right)} \\
& =\underset{\left(b_{m n}\right)}{\operatorname{Sp}}\left\{B_{m n} B_{m n+1}^{*}\right\},
\end{aligned}
$$

where in the final lines we introduce the abbreviated notation for the arising factors, to be called shortly Grassmann factors:

$$
\begin{array}{ll}
A_{m n}=1+a_{m n} \sigma_{m n}, & A_{m+1 n}^{*}=1+t_{m+1 n}^{(1)} a_{m n}^{*} \sigma_{m+1 n}, \\
B_{m n}=1+b_{m n} \sigma_{m n}, & B_{m n+1}^{*}=1+t_{m n+1}^{(2)} b_{m n}^{*} \sigma_{m n+1},
\end{array}
$$

while $S p(\ldots)$ stand for the Gaussian averaging like $d a^{*} d a \mathrm{e}^{a a^{*}}(\ldots)$ and $\int d b^{*} d b \mathrm{e}^{b b^{*}}(\ldots)$. These local averaging symbols, which are even in fermions, are totally commuting with any element of the algebra and can be gathered in one place, forming the symbol of the global Gaussian averaging. The identities (15) can be checked making use of the elementary rules of fermionic integration like (3), taking into account that $\exp \left(a a^{*}\right)=1+a a^{*}$ and $\exp \left(b b^{*}\right)=1+b b^{*}$. Notice that the $m n$ indices in the above Grassmann factors are chosen to be equal to the indices of the spin variables involved in these factors. Thus, it will be easy to control the position of any Grassmann factor with given spin variable among other such factors in their global products.

The idea of the next step is to substitute (15) into
(13) and to eliminate the spin variables in the resulting mixed representation for the density matrix, $Q(\sigma, a)$. To perform the procedure, we have to group together, over the whole lattice, the four factors with the same spin, $A_{m n}, B_{m n}, A_{m n}^{*}, B_{m n}^{*}$, and to average over $\sigma_{m n}= \pm 1$ in each such group of factors, independently. The above four factors come by factorization of the four different bonds attached to a given $m n$ site. In the process of the spin averaging we have to keep these four factors nearby. The separable Grassmann factors, however, are in general neither commuting nor anticommuting with each other, being the superpositions of commuting and anticommuting terms, and it might be difficult, in general, to keep these factors with the same $m n$ nearby over the whole lattice. Therefore, we have to take care of a special 


\section{N. PLECHKO}

ordering for the global products of such factors, in order the elimination of spin variables be really possible. In two dimensions, this problem is solvable [18-20]. Though the individual Grassmann factors are neither commuting nor anticommuting with each other, what can really be used in the ordering arrangements is the property that the doublets like $A_{m n} A_{m+1 n}^{*}$ and $B_{m n} B_{m n+1}^{*}$ representing the local bond weights in (15) can be treated as totally commuting objects, if taken as a whole, under the sign of the Gaussian averaging arising by factorization. Really, the noncommuting terms involved in these doublets, which are linear in fermions, are effectively equal to zero under the averaging, while the remaining terms are even in fermions and are totally commuting. In the reordering transformations we shall apply as well the two ordering principles illustrated below by tutorial examples. The first illustration (linear rearrangement) is:

$$
\begin{aligned}
& \left(\phi_{0} \bar{\phi}_{1}\right)\left(\phi_{1} \bar{\phi}_{2}\right)\left(\phi_{2} \bar{\phi}_{3}\right)\left(\phi_{3} \bar{\phi}_{4}\right) \\
& =\phi_{0}\left(\bar{\phi}_{1} \phi_{1}\right)\left(\bar{\phi}_{2} \phi_{2}\right)\left(\bar{\phi}_{3} \phi_{3}\right) \bar{\phi}_{4}
\end{aligned}
$$

where we simply reread the product joining together the symbols with the same index. The commutation properties of the symbols does not matter at this stage, assuming that we start with a product already ordered as is given in the first line. The second illustration (mirror rearrangement) is:

$$
\begin{aligned}
\left(\phi_{1} \bar{\phi}_{1}\right)\left(\phi_{2} \bar{\phi}_{2}\right)\left(\phi_{3} \bar{\phi}_{3}\right) & =\left(\phi_{1}\left(\phi_{2}\left(\phi_{3} \bar{\phi}_{3}\right) \bar{\phi}_{2}\right) \bar{\phi}_{1}\right) \\
& =\phi_{1} \phi_{2} \phi_{3} \cdot \bar{\phi}_{3} \bar{\phi}_{2} \bar{\phi}_{1}
\end{aligned}
$$

where we assume that the doublets like $\left(\phi_{j} \bar{\phi}_{j}\right)$ are totally commuting with any individual factor from the common set, while the individual factors themselves may be noncommuting. Then we decouple proper and bar factors into separable products.

It is easy to guess that the linear ordering principle (17) is by itself enough to solve the 1D Ising chain via fermionization. This is not the case, however, in two dimensions, where there is a contradiction between preferable $m$-ordering for the horizontal weights and preferable $n$-ordering for the vertical weights, with respect to the linear-ordering rule (17). Therefore, we shall apply first the mirror-ordering principle (18) to factorize a horizontal ladder of the vertical weights, $B_{m n} B_{m n+1}^{*}$, in a horizontal-like fashion with respect to index $m$, with $n$ fixed. This will provide us with an opportunity to introduce properly the remaining horizontal weights at the next stage, so that the spin variables can be finally completely eliminated from the density matrix. With this preliminary notes, let us directly proceed to the ordering arrangements for global products of Grassmann factors arising by factorization of local weights in (15). In transformations from (19) to (20) we omit, for brevity, the signs of the Gaussian averaging introduced by factorization of weights. The totally commuting bond Boltzmann weights are now given by $A_{m n} A_{m+1 n}^{*}$ and $B_{m n} B_{m n+1}^{*}$.

For the first step, let us multiply a subset of vertical weights over $m$, with $n$ fixed. Making use of the mirrorordering rule (18), we write:

$$
\begin{aligned}
& \prod_{m=1}^{L}\left(1+t_{m n+1}^{(2)} \sigma_{m n} \sigma_{m n+1}\right)=\prod_{m=1}^{L} B_{m n} B_{m n+1}^{*} \\
& =\prod_{m=1}^{L} \stackrel{{ }^{m}}{B_{m n}} \cdot \prod_{m=1}^{L} \stackrel{m}{B_{m n+1}^{*}} .
\end{aligned}
$$

In the final expression, there are two $m$-ordered products with $m=1, \ldots, L$ going in the opposite directions (mirror ordering). Already at this stage the ordering is favourable for introducing the horizontal weights $A_{m n} A_{m+1 n}^{*}$ into a one of the $m$-ordered products. This possibility is used below. Meanwhile, let us continue with the vertical weights. Multiplying the above partial products taken as a whole over $n=1, \ldots, L$, with $n$ increasing from left to right, and making use of the linear-ordering rule (17) with respect to index $n$, we write:

$$
\begin{aligned}
& \prod_{n=1}^{L} \prod_{m=1}^{L}\left(1+t_{m n+1} \sigma_{m n} \sigma_{m n+1}\right) \\
& =\stackrel{\stackrel{n}{\longrightarrow}}{\prod_{n=1}^{L}}\left[\prod_{m=1}^{L} \stackrel{\stackrel{m}{B_{m n}}}{\prod_{m=1}^{L}} \stackrel{\stackrel{m}{B_{m n+1}^{*}}}{\stackrel{m}{\longrightarrow}}\right] \\
& =\stackrel{\vec{n}}{\stackrel{\vec{L}}{L}}\left[\prod_{n=1}^{L} \stackrel{{ }^{m}}{B_{m n}^{*}} \cdot \prod_{m=1}^{L} \stackrel{\stackrel{m}{B}_{m n}}{B_{m=1}}\right]
\end{aligned}
$$

When we pass to the last line, it was taken into account that $B_{m L+1}^{*}=1$, since $\sigma_{m L+1}=0$, according to the free-boundary conditions we have assumed above. Respectively, we have corrected the final expression in (20) at the left end, introducing the lacking product of factors with $n=1$, which are of the form $B_{m 1}^{*}=1+t_{m 1} \sigma_{m 1} b_{m 0}^{*}$, where we put $b_{m 0}^{*}=0$. Actually, $B_{m 1}^{*}=1$, with $b_{m 0}^{*}=0$. In this way, the free boundary conditions for spins are now elaborated into the analogous conditions for fermions.

All vertical weights are already involved in the product $(20)$. It remains only to introduce properly the commuting horizontal weights, $A_{m n} A_{m+1 n}^{*}$, into a one of the $m$-ordered products in (20). Evidently, the products of factors $B_{m n}^{*}$ are preferable. Making use of the linear rule (18) for the subsequent transformations with respect to $m$, for the complete density matrix we get: 


$$
\begin{aligned}
& Q(\sigma)=\prod_{n=1}^{L} \prod_{m=1}^{L}\left(1+t_{m n+1} \sigma_{m n} \sigma_{m n+1}\right)\left(1+t_{m+1 n} \sigma_{m n} \sigma_{m+1 n}\right)
\end{aligned}
$$

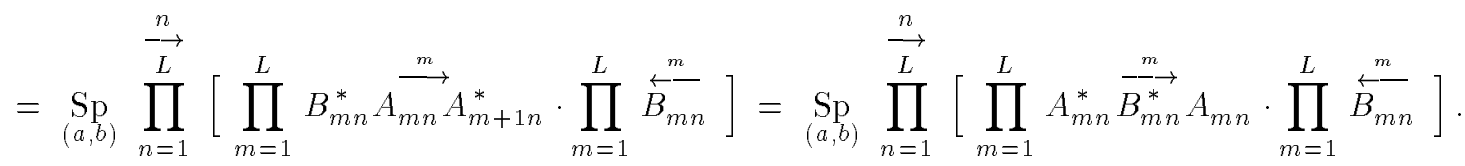

By analogy with the boundary transformations in (20), we eliminate in the final line the extra factors $A_{L+1 n}^{*}=1$, with $\sigma_{L+1 n}=0$, and insert, formally, the lacking factors $A_{1 n}^{*}=1$, assuming $a_{0 n}^{*}=0$. In (21) we also restore the symbol of the diagonal Gaussian averaging arising by factorization of the local weights:

$$
\operatorname{Sp}_{(a, b)}\{\ldots\}=\int \prod_{m=1}^{L} \prod_{n=1}^{L} d a_{m n}^{*} d a_{m n} d b_{m n}^{*} d b_{m n} \exp \sum_{m=1}^{L} \sum_{n=1}^{L}\left(a_{m n} a_{m n}^{*}+b_{m n} b_{m n}^{*}\right)\{\ldots\} .
$$

The expression in the final line of (21) is what we call the mirror-ordered factorized representation for the density matrix. This representation is exact, assuming free-boundary conditions for fermions, $a_{0 n}^{*}=0, b_{m 0}^{*}=0$. The density matrix is now completely prepared for the elimination of the spin variables. The partition function arises by summing over the states $\sigma_{m n}= \pm 1$ at each site in (21).

The averaging over $\sigma_{m n}= \pm 1$ is to be performed at the junction of the two $m$-ordered products in (21), with fixed $n$. This is a step by step procedure. The local averaging at the junction is given by:

$$
\begin{aligned}
& \underset{\left(\sigma_{m n}\right)}{\operatorname{Sp}}\left\{A_{m n}^{*} B_{m n}^{*} A_{m n} B_{m n}\right\}=\frac{1}{2} \sum_{\sigma_{m n}= \pm 1}\left(1+t_{m n}^{(1)} \sigma_{m n} a_{m-1 n}^{*}\right)\left(1+t_{m n}^{(2)} \sigma_{m n} b_{m n-1}^{*}\right)\left(1+\sigma_{m n} a_{m n}\right)\left(1+\sigma_{m n} b_{m n}\right) \\
= & 1+a_{m n} b_{m n}+t_{m n}^{(1)} t_{m n}^{(2)} a_{m-1 n}^{*} b_{m n-1}^{*}+\left(t_{m n}^{(1)} a_{m-1 n}^{*}+t_{m n}^{(2)} b_{m n-1}^{*}\right)\left(a_{m n}+b_{m n}\right)+t_{m n}^{(1)} t_{m n}^{(2)} a_{m-1 n}^{*} b_{m n-1}^{*} a_{m n} b_{m n} \\
= & \exp \left[a_{m n} b_{m n}+t_{m n}^{(1)} t_{m n}^{(2)} a_{m-1 n}^{*} b_{m n-1}^{*}+\left(t_{m n}^{(1)} a_{m-1 n}^{*}+t_{m n}^{(2)} b_{m n-1}^{*}\right)\left(a_{m n}+b_{m n}\right)\right]
\end{aligned}
$$

The result of the averaging is a purely fermionic polynomial, even in the variables, which is equivalent to the Gaussian exponential factor given in the last line. This equivalence can be checked, for instance, by the series expansion of the exponential, taking into account the nilpotent property of fermions. Another way to see this equivalence is explained in Section 6. Let $n$ be fixed, at the junction of the two $m$-ordered products in (21) we just find the four relevant Grassmann factors (23) with the same index $m n$ placed nearby, with $m=L$, given $n$. The local averaging (23) results the Gaussian exponential factor from the last line, which is even in fermions, thus, totally commuting with any element of the algebra. We then remove this commuting Gaussian factor from the junction somewhere to the very left end of the remaining ordered product, and find again at the junction a new set of four neighbouring factors like (23) with the same index $m n$ and the same spin variable, with $m=L-1$, given $n$. We then repeat the same averaging procedure at the junction for $m=L-1$ and then for $m=L-2, \ldots, 1$, for given $n$, and all over again for other values of $n=1, \ldots, L$. The spin variables being completely eliminated, over the whole lattice, the partition appears to be given by the product of the partial Gaussian exponential factors from (23) under the sign of the global Gaussian averaging (22). Thus we come to the result:

$$
\begin{aligned}
Q & =\int \prod_{m=1}^{L} \prod_{n=1}^{L} d b_{m n}^{*} d b_{m n} d a_{m n}^{*} d a_{m n} \exp \left\{\sum _ { m = 1 } ^ { L } \sum _ { n = 1 } ^ { L } \left[a_{m n} a_{m n}^{*}+b_{m n} b_{m n}^{*}\right.\right. \\
& \left.\left.+a_{m n} b_{m n}+t_{m n}^{(1)} t_{m n}^{(2)} a_{m-1 n}^{*} b_{m n-1}^{*}+\left(t_{m n}^{(1)} a_{m-1 n}^{*}+t_{m n}^{(2)} b_{m n-1}^{*}\right)\left(a_{m n}+b_{m n}\right)\right]\right\},
\end{aligned}
$$




\section{N. PLECHKO}

where $a_{0 n}^{*}=0, b_{m 0}^{*}=0$. The partition function is now presented as a fermionic Gaussian integral. This representation is exact. The fermionic integral (24) is completely equivalent to the original expression (13) assuming the free boundary conditions both for spins and fermions.

\section{MOMENTUM-SPACE FERMIONS}

In this section we consider the 2D Ising model settled on the standard homogeneous lattice. The partition function $Q$ can be explicitly evaluated in this case by the transformation to the momentum space for fermions. This results in the Onsager expressions for the partition function and free energy of the standard 2D Ising model. For the homogeneous (though yet anisotropic) lattice, in the Hamiltonian (10) we put: $b_{m n}^{(1)}, b_{m n}^{(2)} \rightarrow b_{1}, b_{2}$, where $b_{1,2}=\beta J_{1,2}$ are the dimensionless coupling constants in the horizontal and vertical directions, respectively. The partition function becomes: $Z=\left(2 \cosh b_{1} \cosh b_{2}\right)^{L^{2}} Q$, with the reduced partition function:

$$
Q=\operatorname{Sp}_{(\sigma)}\left\{\prod_{m=1}^{L} \prod_{n=1}^{L}\left(1+t_{1} \sigma_{m n} \sigma_{m+1 n}\right)\left(1+t_{2} \sigma_{m n} \sigma_{m n+1}\right)\right\}
$$

where $t_{1,2}=\tanh b_{1,2}$. From (24), the same partition function is given by the Gaussian integral:

$$
\begin{aligned}
Q & =\int \prod_{m=1}^{L} \prod_{n=1}^{L} d b_{m n}^{*} d b_{m n} d a_{m n}^{*} d a_{m n} \exp \left\{\sum _ { m = 1 } ^ { L } \sum _ { n = 1 } ^ { L } \left[a_{m n} a_{m n}^{*}+b_{m n} b_{m n}^{*}\right.\right. \\
& \left.\left.+a_{m n} b_{m n}+t_{1} t_{2} a_{m-1 n}^{*} b_{m n-1}^{*}+\left(t_{1} a_{m-1 n}^{*}+t_{2} b_{m n-1}^{*}\right)\left(a_{m n}+b_{m n}\right)\right]\right\}
\end{aligned}
$$

with $a_{0 n}^{*}=b_{m 0}^{*}=0$. The integral (26) is equivalent to (25) for any finite lattice size $L$ under the free boundary conditions. In what follows, however, it will be more suitable to impose in (26) the periodic boundary conditions for fermions, $a_{0 n}^{*}=a_{L n}^{*}, b_{m 0}^{*}=b_{m L}^{*}$, which are the most simple and commonly used boundary conditions in order to pass to the momentum space for a finite discrete lattice. This change of conditions can be viewed as a boundary approximation inessential for infinite lattice, $N=L^{2} \rightarrow \infty$. Finally, we are interested in the free energy per site for infinite lattice. Assuming in (26) the periodic boundary conditions for fermions, we pass to the momentum space by the standard Fourier substitution:

$$
\begin{array}{ll}
a_{m n}=\frac{1}{L} \sum_{p=0}^{L-1} \sum_{q=0}^{L-1} a_{p q} \mathrm{e}^{i \frac{2 \pi}{L} m p+i \frac{2 \pi}{L} n q}, & a_{m n}^{*}=\frac{1}{L} \sum_{p=0}^{L-1} \sum_{q=0}^{L-1} a_{p q}^{*} \mathrm{e}^{-i \frac{2 \pi}{L} m p-i \frac{2 \pi}{L} n q}, \\
b_{m n}=\frac{1}{L} \sum_{p=0}^{L-1} \sum_{q=0}^{L-1} b_{p q} \mathrm{e}^{i \frac{2 \pi}{L} m p+i \frac{2 \pi}{L} n q}, & b_{m n}^{*}=\frac{1}{L} \sum_{p=0}^{L-1} \sum_{q=0}^{L-1} b_{p q}^{*} \mathrm{e}^{-i \frac{2 \pi}{L} m p-i \frac{2 \pi}{L} n q} .
\end{array}
$$

In the momentum space, the integral becomes:

$$
\begin{aligned}
Q & =\int \prod_{p=0}^{L-1} \prod_{q=0}^{L-1} d a_{p q}^{*} d a_{p q} d b_{p q}^{*} d b_{p q} \exp \left\{\sum _ { p = 0 } ^ { L - 1 } \sum _ { q = 0 } ^ { L - 1 } \left[a_{p q} a_{p q}^{*}+b_{p q} b_{p q}^{*}+a_{p q} b_{L-p L-q}\right.\right. \\
& \left.\left.+t_{1} t_{2} \mathrm{e}^{i \frac{2 \pi p}{L}-i \frac{2 \pi q}{L}} a_{p q}^{*} b_{L-p L-q}^{*}+\left(t_{1} \mathrm{e}^{i \frac{2 \pi p}{L}} a_{p q}^{*}+t_{2} \mathrm{e}^{i \frac{2 \pi q}{L}} b_{p q}^{*}\right)\left(a_{p q}+b_{p q}\right)\right]\right\} .
\end{aligned}
$$

where $a_{p q}, a_{p q}^{*}, b_{p q}, b_{p q}^{*}$ are the new variables of the integration. In the above transformation, the orthogonality relations for the Fourier exponents were taken into account:

$$
\frac{1}{L^{2}} \sum_{m=1}^{L} \sum_{n=1}^{L} \exp \left[i \frac{2 \pi}{L} m\left(p \pm p^{\prime}\right)+i \frac{2 \pi}{L}\left(q \pm q^{\prime}\right) n\right]=\delta\left(p \pm p^{\prime} \mid q \pm q^{\prime}\right) \bmod L
$$


where $\delta(p \mid q) \bmod L$ is the Kronecker symbol modulo $L$ in both directions. The fermionic measure in (28) transforms in a trivial way (Jacobian equals to unity) due to the unitarity of the combined Fourier substitution (27), which property follows from (29). Thus we have to evaluate explicitly the momentum-space integral (28).

The fermionic action in the momentum space admits a block-diagonal structure and the integral decouples into a product of low-dimensional integrals over the groups of the variables with momenta $p, q$ and $L-p, L-q$. Since the variables with conjugated momenta $p q$ and $L-p L-q$ are interacting, in order to single out explicitly the true independent subsets of the variables in the action, which will correspond to factorization of $Q$ into the truly independent integral factors, we have to combine together in the $p q$-sum in (28) the terms with conjugated momenta $p, q$ and $L-p, L-q$. Equivalently, the $p q$-sum is to be symmetrized with respect to conjugation $p, q \leftrightarrow L-p, L-q$. After such a symmetrization, the integral (28) factorizes into a product of independent integral factors of the following kind:

$$
\begin{aligned}
Q_{p q}^{2} & =\int d a_{p q}^{*} d a_{p q} d b_{p q}^{*} d b_{p q} d a_{L-p L-q}^{*} d a_{L-p L-q} d b_{L-p L-q}^{*} d b_{L-p L-q} \exp \left[\left(a_{p q} a_{p q}^{*}\right.\right. \\
& \left.+b_{p q} b_{p q}^{*}+a_{L-p L-q} a_{L-p L-q}^{*}+b_{L-p L-q} b_{L-p L-q}^{*}\right)+\left(a_{p q} b_{L-p L-q}+a_{L-p L-q} b_{p q}\right) \\
& +\left(\hat{t}_{1} \hat{t}_{2}^{*} a_{p q}^{*} b_{L-p L-q}^{*}+\hat{t}_{1}^{*} \hat{t}_{2} a_{L-p L-q}^{*} b_{p q}^{*}\right)+\left(\hat{t}_{1} a_{p q}^{*}+\hat{t}_{2} b_{p q}^{*}\right)\left(a_{p q}+b_{p q}\right) \\
& \left.+\left(\hat{t}_{1}^{*} a_{L-p L-q}^{*}+\hat{t}_{2}^{*} b_{L-p L-q}^{*}\right)\left(a_{L-p L-q}+b_{L-p L-q}\right)\right]
\end{aligned}
$$

where we assume abbreviations:

$$
\hat{t}_{1}=t_{1} \mathrm{e}^{i \frac{2 \pi p}{L}}, \quad \hat{t}_{2}=t_{2} \mathrm{e}^{i \frac{2 \pi g}{L}}, \quad \hat{t}_{1}^{*}=t_{1} \mathrm{e}^{-i \frac{2 \pi p}{L}}, \quad \hat{t}_{2}^{*}=t_{2} \mathrm{e}^{-i \frac{2 \pi g}{L}} .
$$

The elementary Gaussian integral (30) can be evaluated in different ways. The straightforward method is to expand the nondiagonal part of the exponential into a series and to integrate step by step over the subsets of the conjugated variables by means of elementary rules like (3). In the advanced version of this method, one makes use of the selection rules for the diagonal Gaussian averages that can be observed in the relations like (15) and (56). Another method is to interpret (30) as determinantal Gaussian integral like (6) with $N=4$. In such representation, one assumes the Gaussian action in the form: $S=a \hat{A} a^{*}$, which is possible, for instance, with the following choice of the conjugated fields:

$$
\begin{aligned}
& a_{1}, a_{2}, a_{3}, a_{4} \leftrightarrow a_{p q}, b_{p q}, a_{L-p L-q}^{*}, b_{L-p L-q}^{*}, \\
& a_{1}^{*}, a_{2}^{*}, a_{3}^{*}, a_{4}^{*} \leftrightarrow a_{p q}^{*}, b_{p q}^{*}, a_{L-p L-q}, b_{L-p L-q} .
\end{aligned}
$$

The integral factor (30) then equals to the determinant of matrix $A$ given explicitly in (33). Thus, we find:

$$
Q_{p q}^{2}=\operatorname{det}\left|\begin{array}{cccc}
1-\hat{t}_{1} & -\hat{t}_{2} & 0 & 1 \\
-\hat{t}_{1} & 1-\hat{t}_{2} & -1 & 0 \\
0 & \hat{t}_{1}^{*} \hat{t}_{2} & -1+\hat{t}_{1}^{*} & \hat{t}_{1}^{*} \\
-\hat{t}_{1} \hat{t}_{2}^{*} & 0 & \hat{t}_{2}^{*} & -1+\hat{t}_{2}^{*}
\end{array}\right| \text {. }
$$

By a straightforward though somewhat lengthy calculation of the above determinant, we arrive to at the following expressions:

$$
\begin{aligned}
Q_{p q}^{2} & =\left(1+\left|\hat{t}_{1}\right|^{2}\right)\left(1+\left|\hat{t}_{2}\right|^{2}\right)-\left(\hat{t}_{1}+\hat{t}_{1}^{*}\right)\left(1-\left|\hat{t}_{2}\right|^{2}\right)-\left(\hat{t}_{2}+\hat{t}_{2}^{*}\right)\left(1-\left|\hat{t}_{1}\right|^{2}\right) \\
& =\left(1+t_{1}^{2}\right)\left(1+t_{2}^{2}\right)-2 t_{1}\left(1-t_{2}^{2}\right) \cos \frac{2 \pi p}{L}-2 t_{2}\left(1-t_{1}^{2}\right) \cos \frac{2 \pi q}{L}
\end{aligned}
$$

To obtain the partition function, $Q$, we have to multiply the factors (34) over all distinct pairs of the conjugated 


\section{N. PLECHKO}

momentum-lattice points $(p, q \mid L-p, L-q)$. That is, if the factor $Q_{p q}^{2}$ with given $p q$ is already included into the product, then the factor $Q_{L-p L-q}^{2}$ is not to be included, and vice versa (notice by the way that $Q_{p q}^{2}=Q_{L-p L-q}^{2}$ ). The above prescription can be readily seen also comparing fermionic measures in (28) and (30). Respectively, if we multiply the factors $Q_{p q}^{2}$ over all the points of the momentum lattice with no restrictions, this will yield squared partition function, $Q^{2}$. Thus, we find:

$$
Q^{2}=\prod_{p=0}^{L-1} \prod_{q=0}^{L-1}\left[\left(1+t_{1}^{2}\right)\left(1+t_{2}^{2}\right)-2 t_{1}\left(1-t_{2}^{2}\right) \cos \frac{2 \pi p}{L}-2 t_{2}\left(1-t_{1}^{2}\right) \cos \frac{2 \pi q}{L}\right] .
$$

In essence, this is equivalent to Onsager's solution for the 2D Ising model on the standard rectangular lattice in a zero magnetic field [1]. The trigonometric product (35) is the exact solution for $Q^{2}$ in the limit $L^{2} \rightarrow \infty$. The correspondent free energy per site readily follows:

$$
-\beta f_{Q}=\left.\frac{1}{L^{2}} \ln Q\right|_{L \rightarrow \infty}=\frac{1}{2} \int_{0}^{2 \pi} \int_{0}^{2 \pi} \frac{d p}{2 \pi} \frac{d q}{2 \pi} \ln \left[\left(1+t_{1}^{2}\right)\left(1+t_{2}^{2}\right)-2 t_{1}\left(1-t_{2}^{2}\right) \cos p-2 t_{2}\left(1-t_{1}^{2}\right) \cos q\right] .
$$

This is the free energy for the reduced partition function, $Q$, while the true free energy per site, for $Z$, is to be recalculated from $Z=\left(2 \cosh b_{1} \cosh b_{2}\right)^{L^{2}} Q$, and we find:

$$
-\beta f_{Z}=\ln 2+\frac{1}{2} \int_{0}^{2 \pi} \int_{0}^{2 \pi} \frac{d p}{2 \pi} \frac{d q}{2 \pi} \ln \left[\cosh 2 b_{1} \cosh 2 b_{2}-\sinh 2 b_{1} \cos p-\sinh 2 b_{2} \cos q\right],
$$

which is Eq. (108) in [1]. An interesting comment by Lars Onsager on the history of his remarkable solution can be seen in [30]. It is not necessary to say that the method we have applied above to obtain (37) significantly differs from the original approach [1].

In conclusion to this section, let us add few remarks on the properties of the 2D Ising model that follow from the exact solution. [As regards the critical behaviour near $T_{c}$, there is no essential difference between (36) and (37) since the factor between $Q$ and $Z$ is nonsingular at all temperatures]. In what follows, we assume ferromagnetic case, $b_{1,2}>0$. From (36), it can be then deduced that the point of phase transition is given by the condition:

$$
1-t_{1}-t_{2}-t_{1} t_{2}=0
$$

where $t_{1}=\tanh b_{1}, t_{2}=\tanh b_{2}$, with $b_{1}=J_{1} / k T, b_{2}=J_{2} / k T$. Equivalently, this condition can be written in the form:

$$
\sinh 2 b_{1} \cdot \sinh 2 b_{2}=1
$$

which rather corresponds to the free energy in the form (37). The condition (38) for $T_{c}$ can be readily recognized from (36) by writing the $p q$-factor under the logarithm in the form:

$$
\begin{aligned}
Q_{p q}^{2} & =\left(1+t_{1}^{2}\right)\left(1+t_{2}^{2}\right)-2 t_{1}\left(1-t_{2}^{2}\right) \cos p-2 t_{2}\left(1-t_{1}^{2}\right) \cos q \\
& =\left(1-t_{1}-t_{2}-t_{1} t_{2}\right)^{2}+4 t_{1}\left(1-t_{2}^{2}\right) \sin ^{2}(p / 2)+4 t_{2}\left(1-t_{1}^{2}\right) \sin ^{2}(q / 2)
\end{aligned}
$$

Near the origin in the momentum space, $p=q=0$, we have:

$$
\begin{aligned}
& Q_{p q}^{2}=\underline{m}^{2}+A_{1} p^{2}+A_{2} q^{2}, \quad A_{1}=t_{1}\left(1-t_{2}^{2}\right), \quad A_{2}=t_{2}\left(1-t_{1}^{2}\right), \\
& \underline{m}=1-t_{1}-t_{2}-t_{1} t_{2} .
\end{aligned}
$$


It is seen that typically the above factor is positive, $Q_{p q}^{2}>0$, with the only exception for the zero-momentum mode factor, $Q_{p=0 q=0}^{2}$, which vanishes at the point (38). Respectively, there is the singularity in the integrand function in (36) at this point as $p, q \rightarrow 0$. This gives evidence for $(38)$ to be the transition point. Because of the $p q$-integration there is no true singularity (infinity) in the free and the internal energy even at the transition point. The specific heat, however, already exhibits the true logarithmic singularity as $T \rightarrow T_{c}$. The specific heat follows from (37) and/or (36) by differentiating twice with respect to the temperature: $C / k=$ $\beta^{2} \partial^{2}\left(-\beta f_{Z}\right) / \partial \beta^{2}, \beta=1 / k T$. Near the critical point, we have:

$$
C / k \simeq A_{c}|\log \tau| \rightarrow \infty, \quad \tau=\left|\frac{T-T_{c}}{T_{c}}\right| \rightarrow 0
$$

where $C / k$ is the dimensionless specific heat, $k$ is Boltzmann's constant. The parameter $A_{c}$ is called the specificheat critical amplitude. The value of $A_{c}$ is the same by approaching $T_{c}$ from above and from below even for the anisotropic lattice, this is a particular feature of the $2 \mathrm{D}$ Ising model. In the isotropic case $\left(t_{1}=t_{2}=\tanh b\right)$ the specific-heat amplitude is a fixed number: $A_{c}=\frac{8}{\pi} b_{c}^{2} \simeq$ 0.495 , where $b_{c}=\frac{1}{2} \ln (1+\sqrt{2}) \simeq 0.441$ is the inverse critical temperature, $b_{c}=J / k T_{c}$. The asymptotes (42) can be derived substituting (41) into (36) and integrating over some area around the origin in the momentum space [notice that $0 \equiv 2 \pi$ ]. This will yield the singular (nonanalytic) part of the free energy, wherefrom (42) immediately follows. The exact expression for specific heat, over the whole temperature range, also follows from (37) by differentiating twice with respect to the temperature. The resulting expression can be simplified to be expressed in terms of the complete elliptic integrals of the first and second kind $[1,9]$. Further simplifications are not possible. The exact expression for $C / k$ again confirms the asymptotes (42).

Below the critical temperature, there is the spontaneous magnetization in the ferromagnetic 2D Ising model given as follows:

$$
M=\left[1-\frac{1}{\sinh ^{2} 2 b_{1} \sinh ^{2} 2 b_{2}}\right]^{1 / 8}
$$

Near the critical point, $M \simeq B \tau^{1 / 8}$, where $\tau$ is temperature deviation from $T_{c}$, cf. (42). Thus, the critical exponent for magnetization at the critical isobar is: $\beta=1 / 8$. The explicit solution for spontaneous magnetization (43) can be derived by different methods. The derivation given by Yang [3] is based on the transfermatrix method. In the combinatorial approach by Montroll, Potts and Ward [5] it is noted that the squared spontaneous magnetization, $M^{2}$, can be obtained as the limiting value of the two-point spin correlation function for remote spins, $\langle\sigma(0) \sigma(R)\rangle=\left\langle\sigma_{m n} \sigma_{m+R n}\right\rangle$, as $R \rightarrow \infty$. This spin correlation function can be expressed in terms of the perturbed partition function with some line of defects connecting the points 0 and $R$ [5]. Therefore, the result for $M^{2}$ can be also obtained starting with the inhomogeneous fermionic expression for the partition function (24). In this scheme, at the final stages of the calculation, when extracting the asymptotes of the resulting Toeplitz determinant for $\langle\sigma(0) \sigma(R)\rangle$, one can follow, in essence, the same lines as in [5]. However, despite the formal simplicity of the expression for $M$ given in (43), the derivation of this result by any known method, including that with Grassmann variables, remains to be several times more complicated as compared with the derivation of the free-energy expression in the correspondent approach. This is yet an unsolved puzzle in the twodimensional Ising model [3]. For related comments also see $[20,25]$. The analysis of more complicated correlation functions also have been performed and contributed much to our knowledge of phase transition in the 2D Ising model $[8,11,31]$.

\section{THE ORDERED PRODUCTS OF GRASSMANN FACTORS AND GAUSSIAN EXPONENTS}

In this section we add few more remarks about the ordered products of Grassmann factors typically arising, as we have seen, by the fermionic interpretation of the $2 \mathrm{D}$ Ising model within the factorization method. Let $L_{1}$ and $L_{2}$ be arbitrary linear forms in Grassmann variables. Then we have:

$$
\left(1+L_{1}\right)\left(1+L_{2}\right)=\mathrm{e}^{L_{1} L_{2}}\left(1+L_{1}+L_{2}\right)
$$

where the nilpotent properties of fermions where taken into account. In the above equation the two Grassmann factors are combined into a one Grassmann factor accompanied by a Gaussian exponential. The resulting identity can be iterated further on, and we find:

$$
\begin{aligned}
& \left(1+L_{1}\right)\left(1+L_{2}\right)\left(1+L_{3}\right) \ldots\left(1+L_{N}\right) \\
& =\left(1+\sum_{i=1}^{N} L_{i}\right) \exp \left(\sum_{1 \leq i<j \leq N} L_{i} L_{j}\right)
\end{aligned}
$$

where $L_{1}, \ldots, L_{N}$ are arbitrary linear forms in Grassmann variables. Let $\sigma_{0}= \pm 1$ be Ising spin, notice that $\sigma_{0}^{2}=1$. Making substitution $L_{j} \rightarrow L_{j} \sigma_{0}$ in (45), we obtain the identity:

$$
\begin{aligned}
& \left(1+L_{1} \sigma_{0}\right)\left(1+L_{2} \sigma_{0}\right)\left(1+L_{3} \sigma_{0}\right) \ldots\left(1+L_{N} \sigma_{0}\right) \\
& =\left(1+\sigma_{0} \sum_{i=1}^{N} L_{i}\right) \exp \left(\sum_{1 \leq i<j \leq N} L_{i} L_{j}\right)
\end{aligned}
$$

The averaging over the spin states then results: 


$$
\begin{aligned}
& \underset{\left(\sigma_{0}\right)}{\operatorname{Sp}_{0}}\left\{\left(1+L_{1} \sigma_{0}\right)\left(1+L_{2} \sigma_{0}\right)\left(1+L_{3} \sigma_{0}\right) \ldots\left(1+L_{N} \sigma_{0}\right)\right\}=\exp \left(\sum_{1 \leq i<j \leq N} L_{i} L_{j}\right), \\
& \underset{\left(\sigma_{0}\right)}{\operatorname{Sp}}(\ldots)=\frac{1}{2} \sum_{\sigma_{0}= \pm 1}(\ldots) .
\end{aligned}
$$

We see that the averaging of a product of any number of the Grassmannian factors like (47) over spin states, $\sigma_{0}= \pm 1$, always results Gaussian fermionic exponential, assuming the Ising spin being the same in all the factors. This property have been used already in the analysis of the 2D Ising models on irregular (in the geometrical sense) planar lattices [24]. The appearance of the Gaussian exponential when we average at the junction in (23) is also evident from (47).

In the same manner, we can elaborate the products of Grassmann factors with different spins, like those appearing in (21):

$$
\begin{aligned}
& \left(1+L_{1} \sigma_{1}\right)\left(1+L_{2} \sigma_{2}\right)\left(1+L_{3} \sigma_{3}\right) \ldots\left(1+L_{N} \sigma_{N}\right) \\
& =\left(1+\sum_{i=1}^{N} L_{i} \sigma_{i}\right) \exp \left(\sum_{1 \leq i<j \leq N} \sigma_{i} \sigma_{j} L_{i} L_{j}\right) .
\end{aligned}
$$

This identity is a generalization (or a particular case) of (45). In Eqs. (44)-(48), it is only important that $L_{1}, \ldots, L_{N}$ are the purely anticommuting symbols, satisfying also the nilpotent property. In principle, in the most general case, we may assume in the above identities $L_{1}, \ldots, L_{N}$ to be arbitrary odd polynomials in Grassmann variables.

The identities like (45) and (48) and related may be of interest also with respect to the 2D Ising model in a nonzero magnetic field. The inclusion of the nonzero magnetic field corresponds to the additional terms ... + $h \sigma_{m n}$ in the hamiltonian (10), which results in the appearance of the additional Boltzmann factors $1+t_{0} \sigma_{m n}$ in the partition function (13) and (21), which are linear in spin variables. Here $t_{0}=\tanh (h)$, where $h=\beta \mathcal{H}$ and $\mathcal{H}$ is a conventional magnetic field in the energy units. Being interested in the effects of small field, $h \rightarrow 0$, one can regard $t_{0} \simeq h$ and $1+t_{0} \sigma_{m n} \simeq 1+h \sigma_{m n}$, as $h \rightarrow 0$. The appearance of such factors prevents the exact solution since the spin variables can not be easily eliminated from the density matrix (21) in this case. Within approximations, however, it can be expected that $h \neq 0$ will make the spins in the ordered products of Grassmann factors like in (21) and (48) to be "frozen", which will induce the nonlocal terms in the action like in (45). With respect to the problem of a non-zero magnetic field in 2D Ising model, and in view of some other potential applications, it may be therefore of interest to consider the nonlocal fermionic action like the one arising in (45) in the momentum space representation.
For visual convenience, let us change the index in the nonlocal fermionic sum of (45) from $i j$ to $\mathrm{mm}^{\prime}$, with $m, m^{\prime}=0,1, \ldots, M-1$. The arising nonlocal Gaussian fermionic action is of the form:

$$
\begin{aligned}
S_{0}(L) & =\sum_{m=0}^{M-2} L_{m}\left(L_{m+1}+\ldots+L_{M-1}\right) \\
& =\sum_{m=0}^{M-2} \sum_{m^{\prime}=m+1}^{M-1} L_{m} L_{m^{\prime}} .
\end{aligned}
$$

It can be expanded over either periodic or aperiodic Fourier exponents. Assuming the aperiodic Fourier substitution:

$$
\begin{aligned}
L_{m} & =\frac{1}{\sqrt{M}} \sum_{p=0}^{M-1} L_{p} \mathrm{e}^{i \frac{2 \pi}{M} m(p+1 / 2)} \\
& =\frac{1}{\sqrt{M}} \sum_{p=0}^{M-1} L_{M-1-p} \mathrm{e}^{-i \frac{2 \pi}{M} m(p+1 / 2)},
\end{aligned}
$$

we find a particularly simple expression:

$$
S_{0}(L)=\sum_{p=0}^{M-1} \frac{L_{p} L_{M-1-p}}{\mathrm{e}^{i \frac{2 \pi}{M} m(p+1 / 2)}-1} .
$$

Assuming the periodic Fourier decomposition:

$$
\begin{aligned}
L_{m} & =\frac{1}{\sqrt{M}} \sum_{p=0}^{M-1} L_{p} \mathrm{e}^{i \frac{2 \pi}{M} m p} \\
& =\frac{1}{\sqrt{M}} \sum_{p=0}^{M-1} L_{M-p} \mathrm{e}^{-i \frac{2 \pi}{M} m p}
\end{aligned}
$$

we obtain a similar though somewhat more sophisticated representation with a special role of the $p=0$ mode:

$$
S_{0}(L)=\sum_{p=1}^{M-1}\left[-L_{0} L_{p}+\frac{\left(L_{p}-L_{0}\right)\left(L_{M-p}-L_{0}\right)}{\mathrm{e}^{i \frac{2 \pi}{M} p}-1}\right]
$$


where $L_{0}=L_{p=0}$. The sums in (51) and (53) can be symmetrized by means of the identity:

$$
\frac{1}{\mathrm{e}^{i p}-1}=\frac{1}{2}\left[\frac{1}{i \tan (p / 2)}-1\right]
$$

In the above identities it is essential that $L_{m}$ are the purely anticommuting fermionic forms in Grassmann variables. There are two remarkable features that can be readily observed in the Fourier sums like (51) and (53). First, we may note that (i) though the action (49) is highly nonlocal in the real space, it becomes diagonal in the momentum space, and the second interesting feature (ii) is the $1 / i p$ singularity in the $p$-mode of the action near $p=0$, as $p \rightarrow 0$. This $1 / i p$ singularity is the essentially fermionic effect, related to the fact that fermions anticommute. The reason for (ii) is that under $p \leftrightarrow-p$ symmetrization fermions just select the skew-symmetric part of the kernel (54), that is, $1 / 2 i \tan (p / 2)$, while the contribution of the symmetric part of that kernel vanishes. The situation will be the opposite for bosons. The analog of the $p \leftrightarrow-p$ symmetrization can as well be performed in (49) in the real space, and we get:

$$
\begin{aligned}
& S_{0}(L)=\frac{1}{2} \sum_{m=0}^{M-1} \sum_{m^{\prime}=0}^{M-1} \epsilon\left(m-m^{\prime}\right) L_{m} L_{m^{\prime}}, \\
& \epsilon\left(m-m^{\prime}\right)=\left\{\begin{array}{rr}
1, & m<m^{\prime} \\
-1, & m>m^{\prime}
\end{array}\right.
\end{aligned}
$$

This is the action of the Gaussian fermionic integral of the second kind with skew-symmetric matrix given in its canonical form, cf. Eq. (7). At the diagonal, effectively, $\epsilon\left(m-m^{\prime}\right)=0$, since $L_{m}^{2}=0$. The properties (i) and (ii) can also be understood, more physically, in the sense that the matrix in (49) is in essence the inverse to $\partial_{m}$, the lat- tice derivative matrix, $\partial_{m} x_{m}=x_{m}-x_{m-1}$. The action $S_{0}(L)$ thus is the sum of terms like $L_{m} \frac{-1}{\partial_{m}} L_{m}$. Since $\partial_{m}$ is diagonal in the momentum space, the nonlocal kernel $1 / \partial_{m}$ also is diagonal in the momentum space. In the low-momentum region (continuum limit) $\partial_{m} \rightarrow i p$, $1 / \partial_{m} \rightarrow 1 / i p$, and $L_{m} \frac{-1}{\partial_{m}} L_{m} \rightarrow L_{p} \frac{1}{i p} L_{-p}$. The Fourier images of lattice $1 / \partial_{m}$ can be seen from (51), (53) and (54). The kernel $\frac{1}{2} \epsilon\left(m-m^{\prime}\right)$ from $(55)$ is the skewsymmetric part of $-1 / \partial_{m}$. For some applications of the above considerations to the 2DIM in a nonzero magnetic field see also the discussion in Section IX.

\section{TWO VARIABLES PER SITE}

In this section we consider some further modifications for the lattice fermionic interpretation of the 2D Ising model, eliminating part of the fermionic variables from the basic Gaussian integral (24) for $Q$. The reduced integral for $Q$ appears to be again Gaussian fermionic integral, but now with only two variables per site, see $(57)$. We intend to apply the identity given below. Let $a, b$ be independent Grassmann variables, then:

$$
\begin{aligned}
\int d b d a \mathrm{e}^{a b+a L_{1}+b L_{2}} & =\int d b d a \mathrm{e}^{a b}\left(1+a L_{1}\right)\left(1+b L_{2}\right) \\
& =\exp L_{2} L_{1}
\end{aligned}
$$

where $L_{1}, L_{2}$ are arbitrary linear forms in some other Grassmann variables, not involved in the integration, but anticommuting with $a, b$. Integrating out from (24) the $a_{m n}, b_{m n}$ fields by means of identity (56), we obtain a reduced Gaussian integral expressed in terms of the remaining variables $a_{m n}^{*}, b_{m n}^{*}$. Let us change the notation for the fields: $a_{m n}^{*}, b_{m n}^{*} \rightarrow c_{m n},-c_{m n}^{*}$, respectively, $d a_{m n}^{*} d b_{m n}^{*} \rightarrow-d c_{m n} d c_{m n}^{*} \rightarrow d c_{m n}^{*} d c_{m n}$. The reduced integral for $Q$ then appears in the form:

$$
Q=\int \prod_{m=1}^{L} \prod_{n=1}^{L} d c_{m n}^{*} d c_{m n} \exp \sum_{m=1}^{L} \sum_{n=1}^{L}\left[c_{m n} c_{m n}^{*}+\left(c_{m n}+c_{m n}^{*}\right)\left(t_{m n}^{(1)} c_{m-1 n}-t_{m n}^{(2)} c_{m n-1}^{*}\right)-t_{m n}^{(1)} t_{m n}^{(2)} c_{m-1 n} c_{m n-1}^{*}\right]
$$

where $c_{m n}, c_{m n}^{*}$ are Grassmann variables, $c_{0 n}=0, c_{m 0}^{*}=0$. The integral (57) is equivalent to (13) and (24), assuming the free boundary conditions in all the cases.

Since the inhomogeneous distribution of the bond coupling parameters is still preserved, all the information on the thermodynamic functions as well as the correlation functions of the 2D Ising model on a rectangular lattice net is still contained in (57). Evidently, the reduction of the number of the variables involved in the integration simplifies the technical aspects of the analysis for regular lattices. This is illustrated below for the standard 2DIM on rectangular lattice. The integral (57) (as well as (24)) may be also of interest with respect to the problem of quenched disorder in the 2D Ising model $[32,33]$. For related discussion also see [26].

For the homogeneous lattice, $t_{m n}^{(1)}, t_{m n}^{(2)} \rightarrow t_{1}, t_{2}$, the integral (57) becomes: 


\section{N. PLECHKO}

$$
\begin{aligned}
Q & =\int \prod_{m=1}^{L} \prod_{n=1}^{L} d c_{m n}^{*} d c_{m n} \exp \sum_{m=1}^{L} \sum_{n=1}^{L}\left[c_{m n} c_{m n}^{*}-t_{1} c_{m-1 n} c_{m n}^{*}-t_{2} c_{m n} c_{m n-1}^{*}\right. \\
& \left.-t_{1} t_{2} c_{m-1 n} c_{m n-1}^{*}+t_{1} c_{m n} c_{m-1 n}+t_{2} c_{m n-1}^{*} c_{m n}^{*}\right] .
\end{aligned}
$$

This integral can be calculated by analogy with (26). We assume again the periodic closing conditions for fermions and pass to the momentum space by Fourier substitution:

$$
c_{m n}=\frac{1}{\sqrt{L^{2}}} \sum_{p=0}^{L-1} \sum_{q=0}^{L-1} c_{p q} \mathrm{e}^{-i \frac{2 \pi p}{L} m+i \frac{2 \pi q}{L} n}, \quad c_{m n}^{*}=\frac{1}{\sqrt{L^{2}}} \sum_{p=0}^{L-1} \sum_{q=0}^{L-1} c_{p q}^{*} \mathrm{e}^{+i \frac{2 \pi p}{L} m-i \frac{2 \pi q}{L} n}
$$

The choice of the signs of $p q$ is here adopted for future convenience in (60). The orthogonality relations (29) are to be taken into account. In the momentum space, the integral (58) becomes:

$$
\begin{aligned}
Q & =\int \prod_{p=0}^{L-1} \prod_{q=0}^{L-1} d c_{p q}^{*} d c_{p q} \exp \left\{\sum _ { p = 0 } ^ { L - 1 } \sum _ { q = 0 } ^ { L - 1 } \left[c_{p q} c_{p q}^{*}\left(1-t_{1} \mathrm{e}^{i \frac{2 \pi p}{L}}-t_{2} \mathrm{e}^{i \frac{2 \pi q}{L}}-t_{1} t_{2} \mathrm{e}^{i \frac{2 \pi p}{L}+i \frac{2 \pi q}{L}}\right)\right.\right. \\
& \left.\left.+t_{1} \mathrm{e}^{i \frac{2 \pi p}{L}} c_{L-p L-q} c_{p q}+t_{2} \mathrm{e}^{i \frac{2 \pi q}{L}} c_{p q}^{*} c_{L-p L-q}^{*}\right]\right\} .
\end{aligned}
$$

Then we have to make the $p, q \leftrightarrow L-p, L-q$ symmetrization of the action in order to single out explicitly the independent subsets of the variables. The integral then decouples into a product of simplest Gaussian fermionic integral factors:

$$
\begin{aligned}
Q_{p q}^{2} & =\int d c_{p q}^{*} d c_{p q} d c_{L-p L-q}^{*} d c_{L-p L-q} \exp \left[c_{p q} c_{p q}^{*}\left(1-t_{1} \mathrm{e}^{i \frac{2 \pi p}{L}}-t_{2} \mathrm{e}^{i \frac{2 \pi q}{L}}-t_{1} t_{2} \mathrm{e}^{i \frac{2 \pi p}{L}+i \frac{2 \pi q}{L}}\right)\right. \\
& +c_{L-p L-q} c_{L-p L-q}^{*}\left(1-t_{1} \mathrm{e}^{-i \frac{2 \pi p}{L}}-t_{2} \mathrm{e}^{-i \frac{2 \pi q}{L}}-t_{1} t_{2} \mathrm{e}^{-i \frac{2 \pi p}{L}-i \frac{2 \pi q}{L}}\right) \\
& \left.+2 i t_{1} \sin \frac{2 \pi p}{L} c_{L-p L-q} c_{p q}+2 i t_{2} \sin \frac{2 \pi q}{L} c_{p q}^{*} c_{L-p L-q}^{*}\right] .
\end{aligned}
$$

This integral factor can be evaluated making use of the elementary rules like (3) and/or (56). Alternatively, if we decide to interpret this integral as the determinant, then we have to present the action in the form: $S=a A a^{*}$, where $A$ is a two by two matrix. This is possible, for instance, assuming the correspondence: $a_{1}, a_{2}, a_{1}^{*}, a_{2}^{*} \leftrightarrow$ $c_{p q}, c_{L-p L-q}^{*}, c_{p q}^{*},-c_{L-p L-q}$. The calculation is very simple in any case, and we find:

$$
\begin{aligned}
Q_{p q}^{2} & =\left|1-t_{1} \mathrm{e}^{i \frac{2 \pi p}{L}}-t_{2} \mathrm{e}^{i \frac{2 \pi q}{L}}-t_{1} t_{2} \mathrm{e}^{i \frac{2 \pi p}{L}+i \frac{2 \pi q}{L}}\right| 2-4 t_{1} t_{2} \sin \frac{2 \pi p}{L} \sin \frac{2 \pi q}{L} \\
& =\left(1+t_{1}^{2}\right)\left(1+t_{2}^{2}\right)-2 t_{1}\left(1-t_{2}^{2}\right) \cos \frac{2 \pi p}{L}-2 t_{2}\left(1-t_{1}^{2}\right) \cos \frac{2 \pi q}{L}
\end{aligned}
$$

The squared partition function follows as the product of factors (62) over the whole momentum-space lattice. The factor in the final line of $(62)$ is the same as in (34). So, we come again to the same results for the partition function and the free energy of the standard 2D Ising model on a rectangular lattice that have been commented already in Section $V$. An interesting new feature in $(62)$ is the trigonometric expression in the first line, wherefrom it is easy to recognize all the possible critical modes (zeroes of $Q_{p q}^{2}$ ) in the ferromagnetic as well as antiferromagnetic regimes. Assuming $p, q$ to be normalized to the $2 \pi$ interval, there are four such possi- ble critical modes: $(p, q)=(0,0),(0, \pi),(\pi, 0),(\pi, \pi)$. In the ferromagnetic case, the only possible critical mode is that with $p=q=0$, and the criticality condition is given by (38) (notice that $0 \equiv 2 \pi$ ). The other three modes, being always positive in the ferromagnetic case $\left(t_{1,2}>0\right)$, define the possible critical points in the antiferromagnetic cases. The above four critical modes and the possible criticality conditions can be recognized already in the momentum-space action (61). With some experience, the above four critical modes can be guessed even from the real-space action (58), without any serious calculation, cf. (63) in Section VIII. For a related 
discussion also see $[20,25]$.

\section{CONTINUUM LIMIT}

Let us now consider the continuum-space limit (low momenta sector) of the exact lattice theory near $T_{c}$. A suitable starting point is the integral (58) for $Q$. In what follows, we assume the homogenous case and ferromagnetic interactions. Let $x_{m n}=c_{m n}, c_{m n}^{*}$, we define lattice derivatives in a natural way: $\partial_{m} x_{m n}=$ $x_{m n}-x_{m-1 n}, \quad \partial_{n} x_{m n}=x_{m n}-x_{m n-1}$. Substituting $c_{m-1 n}=c_{m n}-\partial_{m} c_{m n}, c_{m n-1}^{*}=c_{m n}^{*}-\partial_{n} c_{m n}^{*}$ into (58), we find the action in the form:

$$
\begin{aligned}
S & =\sum_{m n}\left[\underline{m} c_{m n} c_{m n}^{*}-\lambda_{1} c_{m n}^{*} \partial_{m} c_{m n}+\lambda_{2} c_{m n} \partial_{n} c_{m n}^{*}\right. \\
& -t_{1} c_{m n} \partial_{m} c_{m n}+t_{2} c_{m n}^{*} \partial_{n} c_{m n}^{*} \\
& \left.-t_{1} t_{2}\left(\partial_{m} c_{m n}\right)\left(\partial_{n} c_{m n}^{*}\right)\right]
\end{aligned}
$$

with the following set of parameters:

$$
\begin{aligned}
& \underline{m}=\left(1-t_{1}-t_{2}-t_{1} t_{2}\right), \\
& \lambda_{1}=t_{1}\left(1+t_{2}\right), \quad \lambda_{2}=t_{2}\left(1+t_{1}\right) .
\end{aligned}
$$

The lattice action (63) is still the exact expression. In this action one can already distinguish the typical fieldtheoretical like structures, with the mass term and kinetic part. Evidently, the parameter $\underline{m}$ plays the role of mass, while $\lambda_{1}, \lambda_{2}$ and $t_{1}, t_{2}$ are the kinetic coefficients. The critical point can be readily guessed to be $\underline{m}=0$, in agreement with (38). Let us take the formal limit to the continuum space:

$$
\begin{aligned}
& m n \rightarrow x=\left(x_{1}, x_{2}\right), \quad \sum_{m n} \rightarrow \int d^{2} x=\int d x_{1} d x_{2} \\
& \partial_{m} \rightarrow \partial_{1}=\partial / \partial x_{1}, \quad \partial_{n} \rightarrow \partial_{2}=\partial / \partial x_{2} \\
& c_{m n}, c_{m n}^{*} \rightarrow \psi(x), \bar{\psi}(x) \rightarrow \psi, \bar{\psi}
\end{aligned}
$$

The continuum-limit counterpart for the lattice action (63) then appears in the form:

$$
\begin{aligned}
S & =\int d^{2} x\left[\underline{m} \psi \bar{\psi}-\lambda_{1} \psi \partial_{1} \bar{\psi}+\lambda_{2} \psi \partial_{2} \bar{\psi}\right. \\
& \left.-t_{1} \psi \partial_{1} \psi+t_{2} \bar{\psi} \partial_{2} \bar{\psi}\right] .
\end{aligned}
$$

This is the Majorana-like continuum action for twocomponent massive fermions. In the above continuum action we have dropped an interesting second-order momentum term with $\partial_{1} \partial_{2}$. The mass and other parameters are the same as in (64). In presenting the ac- tion in the final form, we have as well applied the rule $\int d^{2} x(a \partial b)=\int d^{2} x(b \partial a)$, where $\partial=\partial_{1}, \partial_{2}$ and $a, b$ are any fermionic fields. This simple rule can be checked by integration by parts, taking into account that fermions anticommute and neglecting the boundary effects. Alternatively, one can check the above rule in lattice interpretation. In $(66)$ the momenta operators $\partial_{1}, \partial_{2}$ in all cases act to the right. The continuum-space action (66) captures the basic features of the exact lattice theory with action (58) in the low-momentum sector near the critical point, which is responsible for the criticalpoint singularities in the thermodynamic functions and the large-distance behaviour of correlations. In the momentum space, this corresponds to approximation like $\mathrm{e}^{i p}-1 \simeq i p, \mathrm{e}^{i q}-1 \simeq i q$, assuming also the ultraviolet cut-off in the momentum integrals, $|p| \leq k_{0}$, with $k_{0}$ of order 1 (or say $\pi / 4$ ) or less.

The Majorana like action (66), however, is not in the canonical form. It can be brought into a canonical form by a suitable linear transformation of the fields, eliminating the undesirable kinetic terms like $\psi_{1} \partial_{1} \bar{\psi}, \psi \partial_{2} \bar{\psi}$. In the canonical form, the 2D Majorana action (66) is given as follows:

$$
\begin{aligned}
S & =\int d^{2} x\left[\bar{m} \psi_{1} \psi_{2}+\psi_{1} \frac{1}{2}\left(\partial_{1}+i \partial_{2}\right) \psi_{1}\right. \\
& \left.+\psi_{2} \frac{1}{2}\left(-\partial_{1}+i \partial_{2}\right) \psi_{2}\right]
\end{aligned}
$$

with the new Majorana components, $\psi_{1}, \psi_{2}$, and the rescaled mass:

$$
\bar{m}=\frac{1-t_{1}-t_{2}-t_{1} t_{2}}{\sqrt{2\left(t_{1} t_{2}\right)_{c}}} .
$$

In order to pass from $(66)$ to $(67)$, we have to transform the fermionic fields and the momenta operators $\partial_{1}, \partial_{2}$ in a suitable way. Here we comment shortly on this transformation. For the first step, let us write the action in the form:

$$
\begin{aligned}
S & =\int d^{2} x\left[\underline{m} \psi_{1} \bar{\psi}_{2}+\psi\left(-\lambda_{1} \partial_{1}+\lambda_{2} \partial_{2}\right) \bar{\psi}\right. \\
& \left.+\frac{t_{1}}{\lambda_{1}} \psi\left(-\lambda_{1} \partial_{1}\right) \psi+\frac{t_{2}}{\lambda_{2}} \bar{\psi}\left(\lambda_{2} \partial_{2}\right) \bar{\psi}\right]
\end{aligned}
$$

By the rescaling of the fields like $\psi \rightarrow \mathrm{e}^{\frac{\mu}{2}} \psi, \bar{\psi} \rightarrow \mathrm{e}^{-\frac{\mu}{2}} \bar{\psi}$, with properly chosen $\mu$, we gain the same action in a slightly more symmetric form:

$$
\begin{aligned}
S & =\int d^{2} x\left[\underline{m} \psi_{1} \bar{\psi}_{2}+\psi\left(-\lambda_{1} \partial_{1}+\lambda_{2} \partial_{2}\right) \bar{\psi}\right. \\
& \left.+\omega_{0}\left(\psi\left(-\lambda_{1} \partial_{1}\right) \psi+\bar{\psi}\left(\lambda_{2} \partial_{2}\right) \bar{\psi}\right)\right]
\end{aligned}
$$

with new kinetic parameter: 


\section{N. PLECHKO}

$$
\begin{aligned}
\omega_{0} & =\sqrt{\frac{t_{1} t_{2}}{\lambda_{1} \lambda_{2}}}=\frac{1}{\sqrt{\left(1+t_{1}\right)\left(1+t_{2}\right)}} \\
& \rightarrow\left(\omega_{0}\right)_{c}=\frac{1}{\sqrt{2}} .
\end{aligned}
$$

Exactly at $T_{c}$, even independently of the rate of the lattice anisotropy, we have $\left(\omega_{0}\right)_{c}=\frac{1}{\sqrt{2}}$, since $\left(1-t_{1}-t-t_{1} t_{2}\right)_{c}=0$. Taking into account that the continuum-limit formulation by itself is reliable only near $T_{c}$, in what follows we put: $\omega_{0}=1 / \sqrt{2}=\left(\omega_{0}\right)_{c}$.

The action (70) is already in a suitable form to be transformed into the canonical Majorana action (67). The general idea is to introduce the new fields by a linear substitution like

$$
\psi=u(\gamma a+\bar{\gamma} \bar{a}), \quad \bar{\psi}=u(\eta a+\bar{\eta} \bar{a})
$$

where $\gamma, \bar{\gamma}, \eta, \bar{\eta}$ are free parameters (four complex numbers) and $a, \bar{a}$ are the new anticommuting components (we shall pass $a, \bar{a} \rightarrow \psi_{1}, \psi_{2}$ at next stages). Substituting (72) into (70), we then look for the uniformization condition that the undesirable terms like $a \partial_{1} \bar{a}, a \partial_{2} \bar{a}$ do not appear in transformed action. In essence, the idea is similar to that of the Bogoliubov transformation in the theories of superfluidity [34] and superconductivity [35]. It appears that the uniformization requirement, in any case, implies the condition $\gamma \bar{\gamma}=\eta \bar{\eta}$ (the above rule $a \partial b=b \partial a$ is not to be forgotten at this stage). We then put $\gamma \bar{\gamma}=\eta \bar{\eta}=1$, assuming the remaining normalization parameter, $u$, to be fixed by the condition $\psi \bar{\psi}=a \bar{a}$, whence $d \bar{\psi} d \psi=d \bar{a} d a$. This is the condition that transformation (72) is canonical, that is, the fermionic measure is unchanged. In this way we come to the uniformization condition in the form:

$$
(\gamma \bar{\eta}+\bar{\gamma} \eta)+2 \omega_{0}=0, \quad \omega_{0}=\sqrt{\frac{t_{1} t_{2}}{\lambda_{1} \lambda_{2}}}
$$

while $u$ is then fixed by the condition:

$$
u^{2}(\gamma \bar{\eta}-\bar{\gamma} \eta)=1
$$

The momenta $\partial_{1}, \partial_{2}$ also will be transformed, in general, under the transformation of the fields like (72). The correspondent relations are not shown, however, since in our particular case, with $\omega_{0}=1 / \sqrt{2}$, this momenta transformation appears to be identical. Assuming $\omega_{0}=1 / \sqrt{2}$, we find that a possible realization for $(72)-(74)$ is the following substitution:

$$
\begin{aligned}
& \psi=\frac{a+\bar{a}}{\sqrt{2 i \sin (\pi / 4)}}, \\
& \bar{\psi}=-\frac{a \mathrm{e}^{i(\pi / 4)}+\mathrm{e}^{-i(\pi / 4)} \bar{a}}{\sqrt{2 i \sin (\pi / 4)}},
\end{aligned}
$$

which corresponds to $\gamma=1, \eta=-\frac{1}{\sqrt{2}}(1+i)$ in $(73)$ and (74). Substituting (75) into (70), the action appears in the form:

$$
\begin{aligned}
S & =\int d^{2} x\left[\underline{m} a \bar{a}+\frac{1}{2} a\left(\lambda_{1} \partial_{1}+i \lambda_{2} \partial_{2}\right) a\right. \\
& \left.+\frac{1}{2} \bar{a}\left(-\lambda_{1} \partial_{1}+i \lambda_{2} \partial_{2}\right) \bar{a}\right]
\end{aligned}
$$

where $a, \bar{a}$ are the new Majorana fields, and $\psi \bar{\psi} \rightarrow a \bar{a}$, $d \bar{\psi} d \psi \rightarrow d \bar{a} d a$. A remarkable feature is that the momenta components $\partial_{1}, \partial_{2}$ in (76) are not effected, they are the same as in the original action (66) and in (70). This feature is provided by a special value of the $\mathrm{ki}$ netic parameter, $\omega_{0} \rightarrow(\omega)_{c}=1 / \sqrt{2}$. The axis in the $d^{2} x \leftrightarrow d^{2} p$ space will be rescaled and rotated, in general, by the uniformization transformation under substitution like $(72)$ with $\omega_{0} \neq 1 / \sqrt{2}$. In fact, the uniformization condition (73) by itself still provides some freedom, corresponding to the gauge rotation of the fields: $a \rightarrow$ $a \mathrm{e}^{i \alpha}, \bar{a} \rightarrow \bar{a} \mathrm{e}^{-i \alpha}$ in (76), or $\psi_{1} \rightarrow \psi_{1} \mathrm{e}^{i \alpha}, \psi_{2} \rightarrow \psi_{2} \mathrm{e}^{-i \alpha}$ in (67), accompanied by the covariant orthogonal rotation of the reference frame of momenta $\lambda_{1} \partial_{1}, \lambda_{2} \partial_{2}$. In (75) and (76) this freedom is fixed in such a way that the axis of the $d^{2} x$ space are not rotated.

Finally, let us rescale the momenta in (76) as follows: $\partial_{1} \rightarrow\left(\lambda_{2} / \lambda_{1}\right)^{1 / 2} \partial_{1}, \partial_{2} \rightarrow\left(\lambda_{1} / \lambda_{2}\right)^{1 / 2} \partial_{2}$, which is canonical transformation $\left(d^{2} x \rightarrow d^{2} x, d^{2} p \rightarrow d^{2} p\right)$. The rescaled momenta are those that finally appear in the canonical action (67). By rescaling, we gain a new overall kinetic factor, let us call it $\omega_{1}$, given by:

$$
\begin{aligned}
\omega_{1} & =\sqrt{\lambda_{1} \lambda_{2}}=\sqrt{t_{1} t_{2}\left(1+t_{1}\right)\left(1+t_{2}\right)} \\
& \rightarrow\left(\omega_{1}\right)_{c}=\sqrt{2\left(t_{1} t_{2}\right)_{c}}
\end{aligned}
$$

it is reasonable to fix $\omega_{1}$ at $T=T_{c}$ as is indicated above. Finally, for the last step, we remove $\omega_{1}$ from the kinetic part by the following rescaling of the fields (first changing the notation for the components) $: a, \bar{a} \rightarrow \psi_{1}, \psi_{2} \rightarrow$ $\psi_{1} / \sqrt{\omega_{1}}, \psi_{2} / \sqrt{\omega_{1}}$, and obtain the Majorana action in the canonical form given in (67). Respectively, the mass $\underline{m}$ from (66) will get renormalized to give the rescaled mass $\bar{m}(68)$.

Up to the stage of this final rescaling of the fields, all the transformations we have applied were canonical, that is, preserving the measure in the integrals of any kind. The last rescaling of the fermionic components, however, is not the canonical transformation, it introduces a factor in the fermionic measure, $d \psi_{2} d \psi_{1} \rightarrow \omega_{1} d \psi_{2} d \psi_{1}$. The correspondent factor then appears in front of the partition function and can be ignored, in essence, by analyzing the critical point. Actually, we have to take care of the invariance of the measure in the $d^{2} x$ space integral (respectively, in the $d^{2} p$ space integral) in the action, but not in the fermionic measure. Notice that the momenta $\partial_{1}, \partial_{2}$ are the same as in the original action (66) up to (76), 
while in (67) there are already the rescaled momenta $\partial_{1}^{\prime}=\left(\lambda_{1} / \lambda_{2}\right)^{1 / 2} \partial_{1}, \partial_{2}^{\prime}=\left(\lambda_{2} / \lambda_{1}\right)^{1 / 2} \partial_{2}$, which is not reflected in the notation of $(67)$. This rescaling, however, is a canonical transformation, $d^{2} x=d^{2} x^{\prime}$, respectively, $d^{2} p=d^{2} p^{\prime}$. This means that we have preserved the normalization of the measure in the momentum space, the singularities near criticality calculated from the canonical action (67) with the mass (68) are expected to be the same, in leading orders, as those provided by the exact lattice theory $(63)$ or its low-momenta approximation (66). The critical-point singularities are commented in Section IX. Meanwhile, let us continue with the canonical Majorana action (67) we have just derived.

The canonical two-component Majorana action (67) can be written as well in matrix notation. Noting that $\bar{m} \psi_{1} \psi_{2}=\frac{1}{2} \bar{m}\left(\psi_{1} \psi_{2}-\psi_{2} \psi_{1}\right)$ and introducing the matrix structure at each space point in the $d^{2} x$ integral, we write:

$$
S_{\text {major }}=\frac{1}{2} \int d^{2} x\left(\begin{array}{c}
\psi_{1} \\
\psi_{2}
\end{array}\right)^{T}\left[\left(\begin{array}{cc}
\partial_{1}+i \partial_{2} & \bar{m} \\
-\bar{m} & -\partial_{1}+i \partial_{2}
\end{array}\right)\right]\left(\begin{array}{l}
\psi_{1} \\
\psi_{2}
\end{array}\right)
$$

where ( $)^{T}$ stands for transposition of spinor. In terms of the standard Pauli matrices, $\sigma_{1}, \sigma_{2}, \sigma_{3}$, the matrix kernel of this action (the 'inverse propagator', or 'equation of motion') can be written in the form: $\left[\bar{m}\left(i \sigma_{2}\right)+\partial_{1}\left(\sigma_{3}\right)+\right.$ $\left.i \partial_{2}(1)\right]$, or in the form: $\left(i \sigma_{2}\right)\left[\bar{m}+\partial_{1}\left(\sigma_{1}\right)+\partial_{2}\left(\sigma_{2}\right)\right]$. Thus, we find:

$$
\begin{aligned}
& S_{\text {major }}=\frac{1}{2} \int d^{2} x \tilde{\Psi}[\bar{m}+\hat{\partial}] \Psi, \quad \tilde{\Psi}=\Psi^{T}\left(i \sigma_{2}\right) \\
& \hat{\partial}=\gamma_{1} \partial_{1}+\gamma_{2} \partial_{2}, \quad \hat{\partial}^{2}=\partial_{1}^{2}+\partial_{2}^{2}
\end{aligned}
$$

with the 2D $\gamma$-matrices $\gamma_{1}=\sigma_{1}, \gamma_{2}=\sigma_{2}$. This is the $2 \mathrm{D}$ Majorana action in the relativistic field-theoretical form. The conjugated Majorana spinors $\tilde{\Psi}$ and $\Psi$ in $(79)$ are built in fact from the same component fields, $\psi_{1}, \psi_{2}$, so they are not the truly independent fields in the path integral. By doubling the number of fermions in the Majorana representation we can pass to the Dirac action with four independent components:

$$
S_{\text {dirac }}=\frac{1}{2} \int d^{2} x \bar{\Psi}(x)[\bar{m}+\hat{\partial}] \Psi(x)
$$

where $\Psi=\left(\psi_{1}, \psi_{2}\right)$ and $\bar{\Psi}=\left(\psi_{1}^{*}, \psi_{2}^{*}\right)^{T}$ are now charged Dirac spinors with four independent anticommuting components: $\psi_{1}, \psi_{2}, \psi_{1}^{*}, \psi_{2}^{*}$. By convention, one can assume $\psi_{1}^{*}, \psi_{2}^{*}$ to be complex conjugates of $\psi_{1}, \psi_{2}$. The propagator $\bar{m}+\hat{\partial}$ in (80) is the same as in (79). To obtain the Dirac action (80), we take two identical copies $S^{\prime}$ and $S^{\prime \prime}$ of the Majorana action (79) and write: $S_{\text {dirac }}=\left(S^{\prime}+S^{\prime \prime}\right)$ majorana. Introducing the new Dirac fields by means of substitution:

$$
\Psi=\frac{1}{\sqrt{2}}\left(\Psi^{\prime}+i \Psi^{\prime \prime}\right), \quad \bar{\Psi}=\frac{1}{\sqrt{2}}\left(\tilde{\Psi}^{\prime}-i \tilde{\Psi}^{\prime \prime}\right)
$$

we obtain the action (80). Mathematically, the transformation from $(79)$ to $(80)$ is in essence the same that we have considered in relation to identity (8) in Section II, which establishes the connection between the fermionic Gaussian integrals of the first and second kind, or between the determinant and the Pfaffian of a skew-symmetric matrix. Notice that the kernel like $\bar{m}\left(i \sigma_{2}\right)+\partial_{1}\left(\sigma_{3}\right)+i \partial_{2}(1)$ from (78) is a skew-symmetric matrix, assuming that $\partial_{1}, \partial_{2}$ are also considered as matrices. The transformations from lattice to continuum in 2DIM are also discussed in $[21,25]$.

\section{CRITICAL-POINT SINGULARITIES}

The field-theoretical formulation for the $2 \mathrm{D}$ Ising model near $T_{c}$ is a suitable representation to discuss, in a simple way, the thermodynamic singularities near the transition point. Assuming that we start with the Dirac interpretation (squared partition function) and noting that in the momentum space $\operatorname{det}(\bar{m}+\hat{\partial})=\bar{m}^{2}+p^{2}$, the singular part of the free energy readily follows in the form:

$$
\begin{aligned}
-\beta f_{\text {sing }} & =\frac{1}{2} \int \frac{d^{2} p}{(2 \pi)^{2}} \ln \left(\bar{m}^{2}+p^{2}\right) \\
& =\frac{1}{8 \pi} \bar{m}^{2} \ln \frac{\mathrm{const}}{\bar{m}^{2}}+(\ldots),
\end{aligned}
$$

where the mass $\bar{m}$ is that given in (68). This is the exact expression for the most singular part of the free energy of the 2DIM in a zero magnetic field $(h=0)$. The same asymptotes follows from the exact solution. Noting that near the critical point $\bar{m} \sim \tau=\left|T-T_{c}\right| / T_{c}$, let us assume $\bar{m}$ for conventional temperature. The internal (average) energy is then given as follows:

$$
(E)_{\bar{m} \text { sing }}=\frac{\partial}{\partial \bar{m}}\left(-\beta f_{\text {sing }}\right)=\int \frac{d^{2} p}{4 \pi^{2}} \frac{\bar{m}}{\bar{m}^{2}+p^{2}}
$$




\section{N. PLECHKO}

$$
=\frac{\bar{m}}{4 \pi} \ln \frac{\text { const }}{\bar{m}^{2}}+(\ldots)
$$

and the dimensionless specific heat is:

$$
\begin{aligned}
(C / k)_{\bar{m} \operatorname{sing}} & =\frac{1}{4 \pi} \ln \frac{\text { const }}{\bar{m}^{2}}+(\ldots) \\
& =\frac{1}{2 \pi} \ln \frac{\text { const }}{|\bar{m}|}+(\ldots)
\end{aligned}
$$

where we put the subscript $\bar{m}$ to remember that the derivatives are taken with respect to the conventional temperature $\bar{m}$. For the isotropic lattice, $\bar{m} \simeq 4\left(b-b_{c}\right) \simeq$ $4 b_{c} \tau$, with $b_{c}=\frac{1}{2} \ln (1+\sqrt{2})$, wherefrom one can recover, for instance, the specific-heat asymptotes (42) with the correct value of the amplitude: $(C / k)_{\operatorname{sing}}=A_{c}|\ln | \tau||$, $A_{c}=(8 / \pi) b_{c}^{2}$

The asymptotes $(82)-(84)$ are to be compared with the hypothetical form of the critical-point singularities in the same functions in a nonzero magnetic field near $T_{c}$, which subject we intend to discuss, in short, in the remaining part of this section. We are interesting merely in what may be the singular behaviour of the specific heat in a nonzero magnetic field along the critical isotherm, that is, when the temperature is fixed exactly at $T_{c}$ and the deviation from the critical point is realized by a small nonzero magnetic field, $h \neq 0$. [For final conclusion, see (89) below]. To start with, let us write the expected form for the singular part of the free energy near the critical isotherm, in the regime of the "strong" magnetic field, $\tau^{15 / 8} \ll h \ll 1:$

$$
-\beta f_{\text {sing }}=\frac{1}{2} \int \frac{d^{2} p}{(2 \pi)^{2}} \ln \left(\bar{m}^{2}+p^{2}+\frac{\lambda^{2}}{p^{2}}\right)+(\ldots),
$$

with $\lambda \propto h M(\tau, h)$, where $M(\tau, h)$ is magnetization, $\tau \rightarrow 0$. More precisely, both $\tau$ and $h$ are assumed to be small, but we are interesting in the situation near the critical isotherm, $\tau=0, h \neq 0$, and introduce infinitesimal deviation from $T_{c}$ with respect to the temperature, $\tau \ll h^{8 / 15}$, merely to perform the differentiation, then we put $\tau \rightarrow 0$. The same form of the free energy can be considered for "weak" field, $h^{8 / 15} \ll \tau$. In this case, however, the choice of the form of $\lambda$ as function of $\tau, h$, due to the strong temperature effects, may be more sophisticated. This may be actual, in particular, in the ordered phase, where the effects of the external field are superimposed on the effects of the inherent molecular field [36].

We comment on singularities that follow from the free energy (85) below, but now let us make few remarks on its origin. A somewhat unusual perturbation term $\lambda^{2} / p^{2}$ which appears in the propagator in (85) is the result of an approximation in the mixed spin-fermion representation for $Q_{\mathrm{h} \neq 0}$. The insertion of the $h>0$ weights like $1+h \sigma_{m n}$ into the factorized density matrix in (21) prevents the exact solution at $h \neq 0$ since the spin variables can not be easily eliminated in this case. We then have elaborated the ordered products of factors in the density matrix (21) into an exponential form, cf. the discussion in Section VI, and applied the simplest approximation of the Hartree-Fock type for the spin subsystem. In particular, this kind of approximation implies $\lambda \sim h M(\tau, h)$. The nonlocal Gaussian exponents, like those considered in Section VI, then appear in action. This, roughly, corresponds to the modification of the Majorana action of the following kind:

$$
S=\frac{1}{2} \int d^{2} x \tilde{\Psi}[\bar{m}+\hat{\partial}+\lambda / \hat{\partial}] \Psi
$$

This form of the action, however, is not to be understood too literally, the less singular $\lambda$-corrections are ignored (or incorporated in $\lambda$ ). There is no essential interference of $\lambda / \hat{\partial}$ with $\bar{m}+\hat{\partial}$ near the line $\tau=0, h \neq 0$ in (85) within given approximations. The main statement is that the free energy appears with the perturbed propagator as is given in (85). Now, let us assume (85) to be true and consider what follows.

The consequences from (85) are interesting. In the strong-coupling regime $\left(\lambda \gg \frac{1}{2} \bar{m}^{2}\right)$ the internal energy per site is given by:

$$
\begin{aligned}
(E)_{\bar{m} \operatorname{sing}} & =\frac{\bar{m}}{4 \pi} \int \frac{p^{2} d p^{2}}{\lambda^{2}+\bar{m}^{2} p^{2}+p^{4}} \\
& +\frac{1}{8} \frac{\partial \lambda}{\partial \bar{m}}+(\ldots) .
\end{aligned}
$$

Respectively, the specific heat at the critical isotherm $(\bar{m} \rightarrow 0)$ appears in the form:

$$
\begin{aligned}
\left(C_{\bar{m}}\right)_{\text {sing }}= & \frac{1}{4 \pi} \int \frac{p^{2} d p^{2}}{\lambda^{2}+\left(p^{2}\right)^{2}}+(\ldots) \\
= & \frac{1}{8 \pi} \ln \frac{\mathrm{const}}{\lambda^{2}}+(\ldots)=\frac{1}{2 \pi}|\ln \sqrt{\lambda}|+(\ldots) \\
& \sqrt{\lambda} \propto h^{8 / 15} \rightarrow 0, \quad \bar{m}=0
\end{aligned}
$$

where $\lambda(0, h) \propto h M(0, h)$, or $\lambda(0, h) \propto h^{16 / 15}$, and we have passed in the final line to $\sqrt{\lambda} \propto h^{8 / 15} \rightarrow 0$ in order to make the amplitude to be equal to that in (84). It is known from scaling and other considerations that $M(0, h) \propto h^{1 / 15}$, wherefrom $\lambda(0, h) \propto h^{16 / 15}$. Comparing (88) with (84), we see that under given approximation the specific heat along the critical isotherm is logarithmic and can be formally recovered from (84) by replacing the thermal mass $\bar{m}=\bar{m}(\tau, 0) \sim \tau$ by the "magnetic mass" $\sqrt{\lambda}(0, h) \sim h^{8 / 15}$. The amplitude in (88) remains the same (with respect to the mass parameters) as in (84). The specific heat (88) is obtained by $\partial^{2} / \partial \bar{m}^{2}$, where $\bar{m}$ is given in (68). Formally, the asymptotes (88) includes the case of the anisotropic lattice as well. For the isotropic lattice, $\bar{m} \simeq 4\left(b-b_{c}\right), b=J / k T$, and we have to multiply the amplitude from (88) by factor $16 b_{c}^{2}$ to obtain the true specific heat along the critical isotherm: 


$$
\begin{aligned}
& (C / k)_{\operatorname{sing}}=\frac{8}{\pi} b_{c}^{2}\left|\ln h^{8 / 15}\right|=E_{c}|\ln h|, \\
& E_{c}=\frac{8}{15} A_{c}=\frac{64}{15 \pi} b_{c}^{2},
\end{aligned}
$$

where $A_{c}=(8 / \pi) b_{c}^{2}$ is the thermal critical amplitude along the critical isobar, $\tau \neq 0, h=0, A_{c}=0.494538589$, while $E_{c}=(8 / 15) A_{c}=0.263753914$ is the amplitude along the critical isotherm, $\tau=0, h \neq 0$, as it appears within given approximation. Here $b_{c}=\frac{1}{2} \ln (1+$ $\sqrt{2})=0.440686793$ is the inverse critical temperature, $b_{c}=J / k T_{c}$. It may be interesting to check (89) by the Monte-Carlo experiments and other numerical methods.

Let us add a remark. The specific heat (88) is obtained, formally, by differentiating with respect to $\bar{m}$ placed in front of the integral in (87) and then taking the limit $\bar{m} \rightarrow 0$. The other corrections are ignored. Evidently, the differentiation with respect to $\bar{m}^{2}$ from the propagator in the integral will yield vanishing contribution as $\bar{m} \rightarrow 0$. As regards the possible contribution from the term $\partial \lambda / \partial \bar{m}$ in the energy (87), the contribution of this term to the specific heat (let $\bar{m} \rightarrow \tau)$ is of order $\frac{\partial^{2}}{\partial \tau^{2}} \lambda(\tau, h) \sim h \frac{\partial^{2}}{\partial \tau^{2}} M(\tau, h)$, but $\frac{\partial^{2}}{\partial \tau^{2}} M(\tau, h) \sim \frac{\partial}{\partial h} \frac{\partial^{2}}{\partial \tau^{2}}\left(-\beta f_{\text {sing }}\right) \sim \frac{\partial}{\partial h} C(\tau, h)$. Taking the limit $\tau \rightarrow 0$, we see that $\frac{\partial^{2}}{\partial \tau^{2}} \lambda \propto h \frac{\partial}{\partial h} \ln h \sim 1$, assuming $C(0, h) \sim \ln h$. So, the logarithmic asymptotes in $(88)$ and (89) is by itself consistent with the less singular contribution via $\lambda(\tau, h)$.

Curiously, we could guess (88) from the most crude phenomenological considerations, simply replacing the "thermal" mass $m_{\tau} \sim \tau$ from (84) by the "magnetic" mass $\bar{m}_{h} \sim h^{8 / 15}$. This replacement can not be done, however, at least in a simple form, in the free energy like (82), since this will yield the expressions with the logarithmic corrections in the functions related to the magnetization at the critical isotherm, which is hardly the case. [For instance, at the critical isobar $M(\tau, 0) \simeq B \tau^{1 / 8}$, and there is no any logarithmic correction. The multiplicative logarithmic corrections are not expected as well neither in $M(0, h)$ nor in $E(0, h)$ ]. The unusual form of the magnetic-field correction $\lambda^{2} / p^{2}$ in the propagator in (85), versus a naive modification of mass term in $\bar{m}^{2}+p^{2}$, is in fact favourable with respect to the known data about the Ising model. Merely, this concerns the absence of the logarithms, observed or expected, in the field derivatives of the free energy.

The 2D Ising model at $T_{c}$ can also be considered in terms of the conformal field theory (CFT) axioms [37-40]. Zamolodchikov [40] has conjectured the existence of the eight masses $m_{i} \sim h^{8 / 15}(i=1,2, \ldots 8)$ in the perturbed CFT assumed to be in the same universality class as the 2DIM at the critical isotherm, $\tau=0, h \neq 0$. A remarkable feature is that the ratios of these masses are predicted from the symmetries as the exact numbers up to the overall normalization constant: $m_{2} / m_{1}=2 \cos \frac{1}{5} \pi, m_{3} / m_{1}=2 \cos \frac{1}{30} \pi$, etc [40]. The nature of these masses from the point of view of the original lattice formulation of 2DIM is yet not well understood. If these masses are thought out as the result of some kind of fine splitting of the $\lambda$ term in the propagator in (85), their effect on the behaviour of the correlations might be different, as compared with the thermal mass effect, since $\lambda^{2}$ is not the same that $\bar{m}^{2}$ in (85). If so, the naive expectation that the asymptotes of the two-point correlation functions will be given, by analogy with thermal decay of correlations, by the sum of the terms like $K_{0}\left(m_{i} R\right)$, where $K_{0}$ is modified Bessel function, may not be the case. It is difficult to make definite predictions, however, at present stage, what may be the modifications. The approximations like (85) seem to be two crude in this respect. It might be conjectured, for instance, that some of the masses (probably all except the lightest or the heaviest one) might have imaginary parts and will then contribute only either more rapidly decaying additive corrections to the leading term (with extra factors $1 / R$ ) or the corrections with the oscillating formfactors (with the same periods $R_{i} \sim m_{i}^{-1}$ as the decay rates in the accompanying exponents) to the term with 'normal' decay, like $K_{0}\left(m_{1} R\right)$, which is what can also be expected from common scaling.

It may be noted that the parameter $\lambda$ in (85) and (86) is rather charge then mass. The free energy in the form (85) might be of interest also at $\mathrm{D} \neq 2$. In principle, taking the free energy in the form (85) as it is, one can try to analyze other thermodynamic functions. However, this will claim for further fine detailing of the meaning of $\lambda$ as a function of both $\tau$ and $h$. In particular, the effects related to the possible spontaneous ordering are to be taken into account properly below $T_{c}$. An interesting feature is that at a special line $\lambda=\frac{1}{2} \bar{m}^{2}$ the free energy (85) reproduces, in essence, the same results (82)-(84) as at $\lambda=0$, that is, at $h=0$. This might be an evidence for the possibility to incorporate the effects of the spontaneous ordering in this scheme. We are going to discuss these subjects in a more detail elsewhere. In fact, the line $\lambda=\frac{1}{2} \bar{m}^{2}$ distinguishes between the weak-field and strong-field regimes, with respect to $\tau$, in the integral (85). At this boundary, $\tau \sim h^{8 / 15}$, this is just what one can expect for this boundary from scaling and other considerations [36].

\section{CONCLUSIONS}

In the above discussion, the two-dimensional Ising model (2DIM) has been treated as a theory of free fermions on a lattice. The anticommuting (Grassmann) variables and integrals were made use of. The fermionization procedure is based on the mirror-ordered fermionic factorization of the density matrix. Following this method, the original spin-variable partition function $Q$ with arbitrary inhomogeneous set of bond coupling parameters was transformed into a Gaussian fermionic integral. The subsequent discussion includes the momentumspace analysis and the exact solution for the standard (translationally invariant) rectangular 2D Ising lattice, the free fermion representation for $Q$ with two variables per site, the Majorana-Dirac field theory interpretation of the 2DIM near $T_{c}$ (continuum limit). The effects of the 


\title{
V. N. PLECHKO
}

long-range fermionic correlations in a nonzero magnetic field and the behaviour of the specific heat along the critical isotherm also have been discussed. Grassmann variables provide a powerful tool to analyze the 2DIM. In physical aspect, it seems to be important to understand better the mechanism of the spontaneous ordering in 2DIM in terms of fermions. The fermionic interpretation of the 2D Ising model provides grounds for this model to be treated in a common range with some other typical models in condensed matter physics and quantum field theory.
[1] L. Onsager, Phys. Rev. 65, 117 (1944).

[2] B. Kaufman, Phys. Rev. 76, 1232 (1949).

[3] C. N. Yang, Phys. Rev. 85, 808 (1952).

[4] M. Kac, J. C. Ward, Phys. Rev. 88, 1332 (1952).

[5] E. W. Montroll, R. B. Potts, J. C. Ward, J. Math. Phys. 4, 308 (1963).

[6] C. A. Hurst, H. S. Green, J. Chem. Phys. 33, 1059 (1960).

[7] T. D. Schultz, D. C. Mattis, E. H. Lieb, Rev. Mod. Phys. 36, 856 (1964).

[8] T. T. Wu, B. M. McCoy, C. A. Tracy, E. Barouch, Phys. Rev. B 13, 316 (1976).

[9] K. Huang, Statistical Mechanics (Wiley, New York, 1963).

[10] H. S. Green, C. A. Hurst, Order-Disorder Phenomena (Interscience, New York, 1964).

[11] B. M. McCoy, T. T. Wu, The Two-Dimensional Ising Model (Harward University Press, Cambridge, MA, 1973).

[12] D. C. Mattis, The Theory of Magnetism II (Springer, Berlin, 1985).

[13] F. A. Berezin, Usp. Matem. Nauk, 24, No. 3, 3 (1969). [Russ. Math. Surveys, 24, No. 3, 1 (1969)].

[14] J. B. Zuber, C. Itzykson, Phys. Rev. D 15, 2875 (1977).

[15] E. Fradkin, M. Srednicki, L. Susskind, Phys. Rev. D 21, 2885 (1980).

[16] S. Samuel, J. Math. Phys. 21, 2806 (1980).

[17] C. Itzykson, Nucl. Phys. B 210, 448 (1982).

[18] V. N. Plechko, Dokl. Akad. Nauk SSSR, 281, 834 (1985). [Sov. Phys. Dokl., 30, 271 (1985)].

[19] V. N. Plechko, Teor. Mat. Fiz. 64, 150 (1985). [Sov. Phys. Theor. Math. Phys. 64, 748 (1985)].

[20] V. N. Plechko, Physica A 152, 51 (1988).

[21] C. Itzykson, J.-M. Drouffe, Statistical Field Theory, Volumes 1 and 2 (Cambridge University Press, Cambridge, 1989).

[22] A. M. Tsvelik, Quantum Field Theory in Condensed
Matter Physics (Cambridge University Press, Cambridge, 1995).

[23] A. I. Bugrij, V. N. Shadura, Phys. Lett. B 150, 171 (1990).

[24] V. N. Plechko, in Selected Topics in Statistical Mechanics, edited by A. A. Logunov et al. (World Scientific, Singapore, 1990), p. 489.

[25] V. N. Plechko, J. Phys. Stud. 1, 554 (1997).

[26] V. N. Plechko, Phys. Lett. A 239, 289 (1998); (E) 245, 563 (1998).

[27] F. A. Berezin, The Method of Second Quantization (Academic Press, New York, 1966).

[28] E. Caianiello, Nuovo Cimento Suppl. 14, 177 (1959).

[29] E. R. Caianiello, Combinatorics and Renormalization in Quantum Field Theory (Benjamin, London, 1973).

[30] L. Onsager, in Critical Phenomena in Alloys, Magnets and Superconductors, edited by R. E. Mills, E. Ascher, R. I. Jaffe (McGraw-Hill, New York, 1971), p. XIXXXIV; p. 3-12.

[31] L. P. Kadanoff, H. Ceva, Phys. Rev. B 3, 3918 (1971).

[32] B. N. Shalaev, Phys. Rep. 237, 129 (1994).

[33] Vik. S. Dotsenko, Usp. Fiz. Nauk 165, 481, (1995) [Sov. Phys. Usp. 38, 310 (1995)].

[34] N. N. Bogolubov, J. Phys. USSR 11, 23 (1947).

[35] N. N. Bogolubov, Zh. Eksp. Teor. Fiz. 34, 58 (1958).

[36] V. N. Plechko, Phys. Lett. A 89, 373 (1982).

[37] Conformal Invariance and Applications to Statistical Mechanics, edited by C. Itzykson, H. Saleur, J.-B. Zuber (World Scientific, Singapore, 1988).

[38] J. L. Cardy, in Fields, Strings and Critical Phenomena, Les Houches, Session XLIX, edited E. Brézin, J. ZinnJustin (Elsevier, Amsterdam, 1989).

[39] A. A. Belavin, A. M. Polyakov, A. B. Zamolodchikov, Nucl. Phys. B 241, 333 (1984).

[40] A. B. Zamolodchikov, Adv. Stud. Pure Math. 19, 641 (1989).

\section{ВІЛЬНІ ФЕРМІОНИ У ДВОВИМІРНІЙ МОДЕЛІ ІЗИНГА}

\author{
В. Н. Плєчко \\ Об'еднаний інститут ядерних досліджень, Лабораторія теоретичнӧ̈ фізики ім. Боголюбова, \\ Дубна, Московська область, 141980, Росіл
}

Переглянуто двовимірну (2D) модель Ізинга як теорію вільних ферміонів на гратці. Обговорення включає процедуру ферміонізапії, що грунтується на дзеркально впорядкованій факторизапіі матрипі густини, зображення статистичної суми гаусівським ферміонним інтегралом, аналіз в імпульсному просторі та результат Онзагера, ефективні теорії поля в гранищі континууму та сингулярности в критичній точщі. Прокоментовано появу далекосяжних ферміонних кореляпій у ненульовому магнетному полі й пове,тінку теплоємности вздовж критичних ізотерм. Приділено увагу виборові раціональних схем розрахунку. 